PII: S0749-6419(98)00050-3

\title{
A FINITE-STRAIN CAM-CLAY MODEL IN THE FRAMEWORK OF MULTIPLICATIVE ELASTO-PLASTICITY
}

\author{
C. Callari, ${ }^{1 *}$ F. Auricchio ${ }^{1}$ and E. Sacco ${ }^{2}$ \\ ${ }^{1}$ Università di Roma Tor Vergata, Dipartimento di Ingegneria Civile, 00133 Roma, Italy \\ ${ }^{2}$ Università di Cassino, Dipartimento di Ingegneria Industriale, 03043 Cassino, Italy
}

(Received in final revised form 26 May 1998)

\begin{abstract}
The present work discusses a finite-strain plasticity model for soft clays. To motivate such a model, the infinitesimal-strain assumption is shown to be inadequate for the constitutive description of soft clays. Hence, assuming the multiplicative elasto-plastic decomposition of the deformation gradient, a finite-strain Cam-clay model is presented. In particular, with respect to the original Cam-clay formulation, this model improves the description of the isotropic compression behaviour as well as of the elastic shearing response. The constitutive laws are discussed and their implications are pointed out. The physical meaning of the model parameters is carefully addressed. Finally, the ability to properly match some experimental results available in the literature is assessed. (C) 1998 Elsevier Science Ltd. All rights reserved
\end{abstract}

Key words: B. elasto-plasticity, B. finite strains, B. geomaterials, A. Cam-clay, B. normal-consolidated clays.

\section{INTRODUCTION}

The constitutive behavior of geomaterials can be effectively modelled in the framework of the elasto-plastic theory, as suggested by the main aspects of their mechanical response, such as strong non-linearity, irreversibility, pressure dependence, shear induced contractancy or dilatancy, volumetric hardening and softening. As a matter of fact, the theory of plasticity is adopted for the solution of the simplest and most frequent geotechnical engineering problems, such as bearing capacity of foundations, stability of retaining structures, slope stability, etc.

It is common practise to indicate the works of Coulomb (1776) and Rankine (1857) as the first applications of plasticity to geomechanics. Although these works are dated more than a century ago, the development of effective elasto-plastic laws for geomaterials is relatively recent. In fact, it has been strongly delayed by the high computational cost and the uncertainties in the material parameters. Hence, only in the last decades, along with the significative improvement of both computational methods and experimental techniques, have important results been obtained in the constitutive modelling of soils and rock.

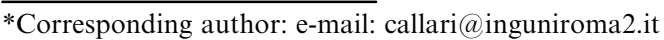


A major aspect of geomaterial mechanical response is the pressure dependence. In the elasto-plasticity framework, this feature can be modelled assuming that the deviatoric section of the yield surface increases with compression. These "conical" yield surfaces were proposed by Drucker and Prager (1952), by Drucker (1953) and by Shield (1955). However, these classical yield criteria do not predict the development of inelastic strains under isotropic compression; furthermore, the assumption of an associative flow rule leads to an overestimation of the dilatant behavior of soils. To overcome the first limit, a conical yield surface closed by a cap along the hydrostatic axis (Cap-model) was proposed by Drucker et al. (1957). The description of the soil dilatant behavior was improved with the Cam-clay model, developed in Cambridge during the '60s (Roscoe and Schofield, 1963). In fact, this model distinguishes between the yield surface and the surface representing the "ultimate", condition of material. The latter is a conical surface; it is called "critical" and it represents the soil states characterized by nil increments of the volumetric plastic strain.

Most of the large number of elasto-plastic models proposed for clays in the last three decades can be considered as improvements of the Cam-clay (overviews can be found in Wroth and Houlsby, 1985; Gens and Potts, 1988). In particular, the "Modified Cam-clay" model (Roscoe and Burland, 1968) differs from the original formulation for the shape of the yield curve and it has proven to be quite accurate in predicting the behavior of soft clays under quasi-static and monotonic loading conditions (e.g. see Wroth, 1975; Wood, 1990). Moreover, it is based on few parameters which can be obtained from conventional laboratory tests (Schofield and Wroth, 1968; Atkinson and Bransby, 1978). These aspects make the Modified Cam-clay frequently adopted for research and design purposes, also in fields involving materials different than soils (e.g. see Saada et al., 1996; Martin et al., 1997). However, the isotropic compression constitutive relations assumed in the original and in the Modified Cam-clay models lead to the prediction of some physically unacceptable behaviors (Hashiguchi, 1995). Moreover, the Cam-clay and the Modified Camclay assumption of an infinite elastic shear modulus is in contrast with the experimental response of clays (Wroth and Houlsby, 1985); similarly, unrealistic responses are obtained using a constant finite modulus, as proposed by several authors to slightly improve predictions and to facilitate the numerical implementation. As an alternative, a shear modulus depending on pressure is frequently assumed; however, the way this dependence is expressed leads to a non-conservative stress-strain relation (Zytinsky et al., 1978).

On the other hand, it can be observed that a finite-strain approach is required for a correct analysis of some frequent geotechnical problems, such as the tilting of towers or the stability of slopes subjected to large movements. In spite of these considerations, almost the totality of the constitutive models proposed for clays are formulated within infinitesimal deformation hypothesis.

Simo and Meschke (1993) proposed a finite-strain Cam-clay model based on the traditional linear $v-\ln p$ relation and a constant elastic shear modulus. The need of an improved description of the response to isotropic compression and elastic shearing has motivated the recent research on a finite-strain extension of the Cam-clay model (Borja and Tamagnini, 1995, 1998; Callari et al., 1996, 1997).

In the following, the constitutive laws of Cam-clay and Modified Cam-clay are recalled and briefly discussed (Section II). Particular emphasis is placed on the discussion of the adopted isotropic compression constitutive relations (Section III). Their main limits are pointed out and some alternative relations proposed in the literature are discussed. 
Furthermore, the infinitesimal-strain hypothesis is shown to be inadequate to the constitutive modelling of soft clays.

Hence, a finite-strain Cam-clay model is formulated within a multiplicative elastoplastic framework (Section IV.2). In particular, the model is based on: (i) an elastic strain energy function with a form similar to the one proposed by Houlsby (1985); (ii) a convex yield function assigned in terms of true stresses and true internal variables and resembling the Modified Cam-clay yield criterion. It is proven that this combination is acceptable from a formal and a physical point of view; in fact, the resulting model does not violate the basic thermodynamic laws and leads to a realistic description of soil behavior.

Section V presents a detailed discussion of several aspects of the model. The attention is focused on the isotropic compression behavior; in particular, the pressure-volume laws in terms of Kirchhoff stress are compared with the analogous relations in terms of Cauchy stress. Starting from this comparison, the "Kirchhoff" model parameters are recovered from the "Cauchy" ones and some considerations on the elasto-plastic decomposition of volumetric strain are reported.

Then, the rate form of the elastic law is recovered and some limits of the model applicability are pointed out. Furthermore, the setting of material parameters and initial conditions is discussed.

Finally, the model ability to reproduce some experimental data available in the literature is investigated in Section VI. In particular, using the implicit integration procedure presented in (Callari et al., 1998), numerical simulations of laboratory tests are carried out and the model predictions are compared with some experimental results reported in (AlTabbaa, 1987).

Throughout this paper, strains and stresses are assumed positive in the case of compression. A standard notation is adopted to indicate the euclidean space $\left(R^{3}\right)$, the space of positively defined symmetric tensors $\left(\mathrm{Sym}^{+}\right)$, the euclidean norm $(\|\cdot\|)$, the trace of a tensor $(\operatorname{tr}(\cdot))$, the symmetric part of a tensor $(\operatorname{sym}(\cdot))$, the deviatoric part of a tensor $(\operatorname{dev}(\cdot))$, the material gradient $(D \cdot)$, the second order (I) and the fourth order (I) identity tensors.

\section{CAM-CLAY AND MODIFIED CAM-CLAY}

The Cam-clay and the Modified Cam-clay elasto-plastic models are both formulated within the infinitesimal-strain hypothesis. In particular, they are based on the classical additive elasto-plastic decomposition of the infinitesimal strain tensor $\varepsilon=\varepsilon^{e}+\varepsilon^{p}$. The formulation of both models is briefly discussed in this Section.

For future developments, the definition of the specific volume is recalled:

$$
v:=\frac{V}{V_{s}}
$$

where $V$ and $V_{s}$, are the current volume of the whole soil and of the solid phase, respectively.

\section{II.1. Elastic stress-strain law}

Both the Cam-clay and Modified Cam-clay adopt a nonlinear elastic law expressed in rate-form as: 


$$
\dot{\boldsymbol{\sigma}}=\left[K \mathbf{I} \otimes \mathbf{I}+2 \mu\left(I-\frac{1}{3} \mathbf{I} \otimes \mathbf{I}\right)\right] \dot{\boldsymbol{\varepsilon}}^{e}
$$

where $K$ and $\mu$ are the bulk and the shear modulus, respectively. In particular, $K$ is a function of the pressure $p:=\frac{1}{3} \operatorname{tr}(\sigma)$ and of the specific volume $v$ :

$$
K=\frac{p v}{k}
$$

with $k$ a dimensionless material constant.

\section{II.2. Yield criterion}

The following yield criteria are assumed:

$$
\begin{aligned}
& f\left(\boldsymbol{\sigma}, p_{c}\right)=q+M p \ln \left(\frac{p}{p_{c}}\right)=0 \text { in Cam-clay } \\
& f\left(\boldsymbol{\sigma}, p_{c}\right)=\frac{q^{2}}{M^{2}}+p\left(p-p_{c}\right)=0 \text { in Modified Cam-clay }
\end{aligned}
$$

where $q:=\sqrt{3 / 2}\|\operatorname{dev}(\sigma)\|$, the quantity $M$ is the slope of the critical state line (CSL) in the $p-q$ plane and $p_{c}$ is the stress-like hardening variable, called preconsolidation pressure.

The Cam-clay yield curve is non-smooth (Fig. 1(a)) and overestimates the deviatoric plastic strain increments, especially for small values of the ratio between octahedral tension and pressure. The elliptical yield curve adopted in the Modified Cam-clay (Fig. 1(b)) improves the deviatoric plastic strain predictions (Roscoe and Burland, 1968).

Figures 2 and 3 show the Modified Cam-clay yield surface in the principal stress space and in the $p-q-v$ space, respectively. In the second representation, the trace of the yield surface on the $p-v$ plane is the normal-consolidation line (NCL), whose physical meaning is explained in Section III.

\section{II.3. Flow rule}

Both models adopt an associative flow rule. For instance, in the Modified Cam-clay the plastic strain increment is expressed as:

(a)

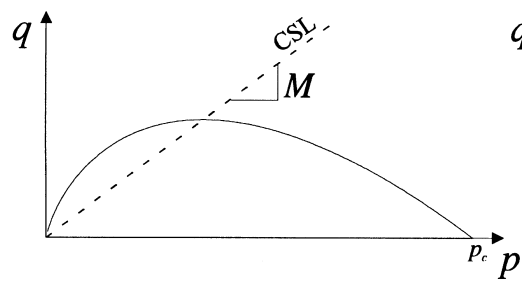

(b)

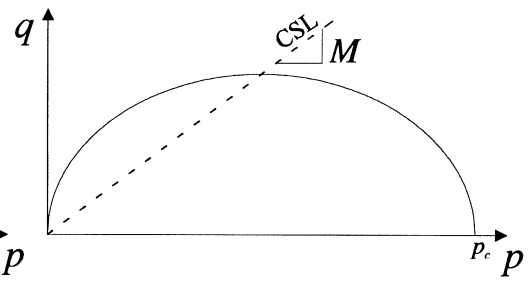

Fig. 1. Cam-clay (a) and Modified Cam-clay (b) yield curves in the $p-q$ plane. 


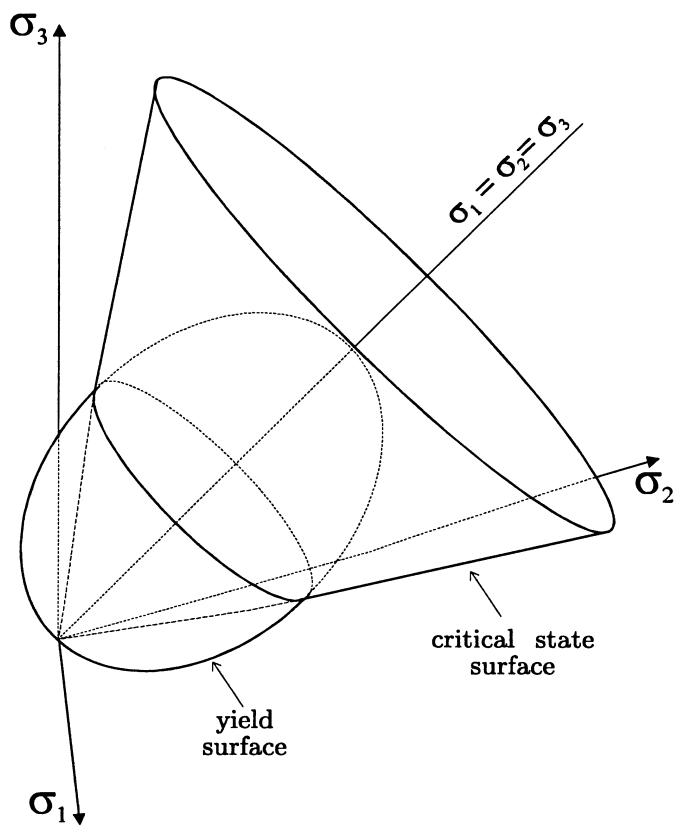

Fig. 2. Modified Cam-clay yield surface and Critical State surface in the principal stress space $\left(\sigma_{1}, \sigma_{2}, \sigma_{3}\right)$.

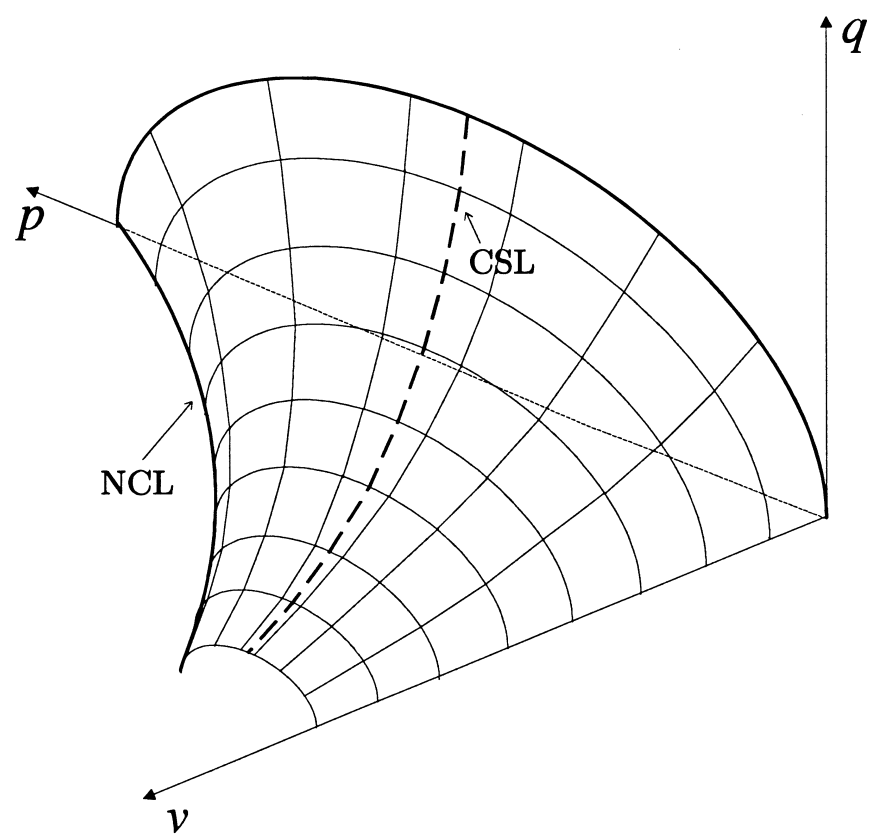

Fig. 3. Modified Cam-clay yield surface in the $p-q-v$ space. 


$$
\dot{\boldsymbol{\varepsilon}}^{p}=\dot{\gamma} \frac{\partial f\left(\boldsymbol{\sigma}, p_{c}\right)}{\partial \boldsymbol{\sigma}}=\dot{\gamma}\left[\frac{1}{3}\left(2 p-p_{c}\right) \mathbf{I}+\frac{3}{M^{2}} \operatorname{dev}(\boldsymbol{\sigma})\right]
$$

where $\dot{\gamma}$ is the plastic consistency parameter.

\section{II.4. Hardening law}

The volumetric hardening law is expressed in rate form as:

$$
\dot{p}_{c}=p_{c} \frac{v}{\lambda-k} \dot{\varepsilon}_{v}^{p}
$$

where $\lambda$ is a material constant and $\dot{\varepsilon}_{v}^{p}$ is the plastic part of the volumetric strain increment $\dot{\varepsilon}_{v}:=\operatorname{tr}(\dot{\varepsilon})$

Remark II.1: In the original works (Roscoe and Schofield, 1963; Roscoe and Burland, 1968), no elastic shear strain is assumed, which corresponds to set an infinite shear modulus $\mu$ in the stress-strain law eqn (2.2). However, not only is this assumption unrealistic, but it also makes difficult the numerical implementation of the model. Hence, two alternative variations of the elastic law are usually adopted.

The first one considers a constant finite value for the elastic shear modulus $\mu$, however, this choice is not in a good agreement with the observed soil behavior (e.g. see Wroth and Houlsby, 1985). Furthermore, for small values of pressure, negative values of the Poisson's ratio are obtained, which is physically unacceptable.

The second variation of the elastic law assumes a fixed value for the Poisson's ratio $v$ (e.g. see Borja and Lee, 1990; Borja, 1991; Hashash and Whittle, 1992; Groen, 1995; Groen et al., 1995), returning the following dependence of the shear modulus on the pressure:

$$
\mu=\frac{3(1-2 v)}{2(1+v)} K=\frac{3(1-2 v)}{2(1+v)} \frac{p v}{k}
$$

Although this approach seems more realistic then the first one, it leads to a nonconservative stress-strain relation (Zytinsky et al., 1978).

\section{ISOTROPIC COMPRESSION CONSTITUTIVE RELATIONS}

III.1. The $v-\ln \mathrm{p}$ relations

Both the original and the Modified Cam-clay models describe the isotropic compression response through linear relations between the specific volume $v$ and the logarithm of the pressure $p$. These relations can be expressed as follows (Fig. 4):

$$
\begin{aligned}
& v=v_{p}-k \ln \left(\frac{p}{p_{0}}\right) \quad \text { in the elastic range } \\
& v_{c}=v_{c 0}-\lambda \ln \left(\frac{p_{c}}{p_{c 0}}\right) \quad \text { in the elasto-plastic range }
\end{aligned}
$$




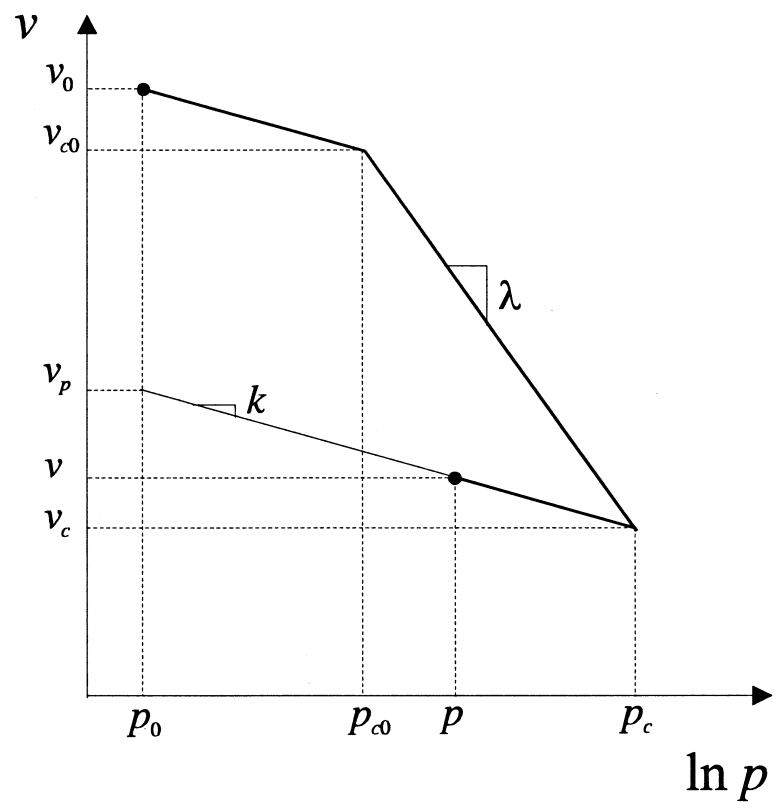

Fig. 4. Logarithmic relations between pressure $(p)$ and specific volume $(v)$.

where $v_{p}$ is the specific volume obtained by unloading the current configuration $(p, v)$ to the initial pressure $p_{0} ; k$ and $\lambda$ are the slopes, in the $v-\ln p$ plane, of the over-consolidation lines (OCL) and of the normal-consolidation line (NCL), respectively. The sets $\left(p_{c 0}, v_{c 0}\right)$ and $\left(p_{c}, v_{c}\right)$ are the pressure and the specific volume pertaining to the initial and the current yielding state, respectively. Some of the consequences relative to the adoption of $v-\ln p$ relations are now investigated.

Using Fig. 4, it is easy to verify the following relation:

$$
v_{c}-v_{c 0}=v_{p}-v_{0}-k \ln \left(\frac{p_{c}}{p_{c 0}}\right)
$$

where $v_{0}$ is the initial specific volume. Substitution of this relation in the normal consolidation line equation (3.2) leads to:

$$
v_{p}-v_{0}-k \ln \left(\frac{p_{c}}{p_{c 0}}\right)=-\lambda \ln \left(\frac{p_{c}}{p_{c 0}}\right)
$$

In clayey materials the solid phase compressibility is usually negligible in comparison with the overall one; hence, the volume of the solid phase can be assumed constant $\left(V_{s} \cong V_{s 0}\right)$. According to this hypothesis and recalling eqn (2.1), the initial and the current volume can be expressed as $V_{0}=v_{0} V_{s 0}$ and $V=v V_{s 0}$, respectively. Therefore, the volumetric strain can be computed as:

$$
E_{v}:=-\frac{V-V_{0}}{V_{0}}=-\frac{v-v_{0}}{v_{0}}
$$


This strain can be decomposed into an elastic and a plastic part according to the additive formula $E_{v}=E_{v}^{e}+E_{v}^{p}$, where:

$$
E_{v}^{e}:=-\frac{v-v_{p}}{v_{0}} \quad E_{v}^{p}:=-\frac{v_{p}-v_{0}}{v_{0}}
$$

Combination of eqn (3.6) with eqns (3.1) and (3.4) leads to the following stress-strain relations in rate form:

$$
\dot{E}_{v}^{e}=\frac{k}{v_{0}} \frac{\dot{p}}{p} \quad \dot{E}_{v}^{p}=\frac{\lambda-k}{v_{0}} \frac{\dot{p}_{c}}{p_{c}}
$$

It must be observed that these constitutive equations are obtained from the pressurevolume relations (3.1-3.2) using the definition of the volumetric strain $E_{v}$ (3.5) and relations (3.6). However, definition (3.5) is rarely used in the geotechnical literature. In fact, the volumetric strain increment is usually defined as $\dot{\theta}=-\dot{v} / v$ (e.g. Schofield and Wroth, 1968; Atkinson and Bransby, 1978; Wood, 1990), whose integration gives the logarithmic volumetric strain:

$$
\theta=-\ln \frac{v}{v_{0}}
$$

Hence, starting from the incremental elasto-plastic decomposition $\dot{\theta}=\dot{\theta}^{e}+\dot{\theta}^{p}$, the following constitutive equations are obtained in rate form (Schofield and Wroth, 1968; Atkinson and Bransby, 1978):

$$
\dot{\theta}^{e}=\frac{k \dot{p}}{v p} \quad \dot{\theta}^{p}=\frac{\lambda-k}{v} \frac{\dot{\theta}_{c}}{p_{c}}
$$

Relations (3.9) are usually preferred to constitutive equations (3.7). In fact, in the Camclay and Modified Cam-clay models, the elastic bulk modulus expression (2.3) and the hardening law (2.7) are given, respectively, by:

$$
\dot{\varepsilon}_{v}^{e}=\frac{k}{v} \frac{\dot{p}}{p} \quad \dot{\varepsilon}_{v}^{p}=\frac{\lambda-k}{v} \frac{\dot{p}_{c}}{p_{c}}
$$

which are (3.9), rewritten in the infinitesimal-strain hypothesis:

$$
\theta:=-\ln \frac{v}{v_{0}} \cong \operatorname{tr}(\varepsilon)=: \varepsilon_{v}
$$

Remark III.1: In the material response predicted by the pressure-volume relations (3.13.2) there are some physically unacceptable aspects (Hashiguchi, 1995); for instance, for high pressure values, negative specific volumes can be reached.

Moreover, according to eqn (3.10), both elastic and elasto-plastic tangent compressibilities decrease as the current specific volume $v$ increases; this feature is unrealistic, because the soil deformability increases with decreasing density ( $v$ increasing). 
Furthermore, the presence of $v$ in $\left(3.10_{1}\right)$ makes the elastic response to depend on the plastic volumetric strain $\varepsilon_{v}^{p}$; the thermodynamic consistency of this elasto-plastic coupling is not a priori verified.

The volumetric strain $\theta$ eqn (3.8) is usually preferred to $E_{v}$ eqn (3.5), as the corresponding stress-strain eqn (3.9) better fit experimental data. This result points out that the infinitesimal-strain hypothesis is inadequate for the constitutive modelling of soft clays, as shown in the following.

Using a standard notation, let a deformation $\varphi: \mathcal{B} \rightarrow R^{3}$ be considered, where $\mathcal{B} \subset R^{3}$ is the body reference configuration. Hence, $\mathbf{x}:=\varphi(\mathbf{X})$ is the position of a material point $\mathbf{X} \in \mathcal{B}$ and $\mathbf{F}(\mathbf{X}):=\partial \varphi(\mathbf{X}) / \partial \mathbf{X}$ is the deformation gradient tensor. It can be shown that $J:=\operatorname{det}[\mathbf{F}]$ measures the current volume for unit volume in the reference configuration, i.e. $J=V / V_{0}$. The displacement associated to $\varphi$ is defined as $\mathbf{u}(\mathbf{X}):=\varphi(\mathbf{X})-\mathbf{X}$ and its gradient is $D \mathbf{u}=\partial \mathbf{u} / \partial \mathbf{X}=\mathbf{F}-\mathbf{I}$. A deformation $\varphi$ is called infinitesimal when its displacement gradient is "small" i.e. when infinitesimals of order higher than:

$$
\omega:=\|\mathbf{F}-\mathbf{I}\|=\|D \mathbf{u}\|
$$

can be neglected in the description of $\varphi$ (Gurtin, 1981).

Consistently with definition (3.12), the volumetric strain can be considered as a "smallness" parameter:

$$
\omega_{v}:=\operatorname{det}[\mathbf{F}]-\operatorname{det}[\mathbf{I}]=J-1=\frac{V-V_{0}}{V_{0}}=:-E_{v}
$$

Neglecting the solid phase compressibility, the parameter $\omega_{v}$ can be rewritten as: $\omega_{v}=\left(v-v_{0}\right) / v_{0}$. The following Taylor series expansion can now be considered:

$$
\ln v=\ln v_{0}+\frac{v-v_{0}}{v_{0}}+O\left(\omega_{v}^{2}\right)
$$

where $O\left(\omega_{v}^{2}\right)$ are the infinitesimals of order higher than $\omega_{v}$. In the infinitesimal-strain hypothesis, the terms $O\left(\omega_{v}^{2}\right)$ can be neglected, leading to:

$$
\ln \frac{v}{v_{0}} \cong \frac{v-v_{0}}{v_{0}}
$$

i.e. $\theta \cong E_{v}$.

Hence, it might be concluded that, if infinitesimal-strain hypothesis were adequate, the constitutive equations obtained from the adoption of $E_{v}$ and of $\theta$ should be equivalent. On the contrary, as already pointed out, the mechanical response of clays is better described by relations (3.9) than by eqn (3.7).

This shows that the approximation $\theta \cong E_{v}$ is in general not acceptable, i.e. $O\left(\omega_{v}^{2}\right)$ terms are not negligible. Therefore, the infinitesimal-strain hypothesis is inadequate for an effective constitutive modelling of soft clays. 


\section{III.2. The $\ln v-\ln p$ relations}

To overcome the shortcomings of the $v-\ln p$ equations (see Remark 3.1), Hashiguchi and Ueno (1977), Butterfield (1979) and Hashiguchi (1995) propose to adopt $\ln v-\ln p$ linear relations:

$$
\begin{aligned}
& \ln \left(\frac{v}{v_{p}}\right)=-k^{*} \ln \left(\frac{p}{p_{0}}\right) \quad \text { in elastic range } \\
& \ln \left(\frac{v_{c}}{v_{c 0}}\right)=-\lambda^{*} \ln \left(\frac{p_{c}}{p_{c 0}}\right) \quad \text { in elasto-plastic range }
\end{aligned}
$$

where $k^{*}$ and $\lambda^{*}$ are the slopes, in the $\ln v-\ln p$ plane, of OCL and NCL, respectively. From eqns (3.16) and (3.17) the following relation is obtained:

$$
\ln \left(\frac{v_{p}}{v_{0}}\right)=-\left(\lambda^{*}-k^{*}\right) \ln \left(\frac{p_{c}}{p_{c 0}}\right)
$$

which is analogous to eqn (3.4). Recalling definition (3.8), the elastic and plastic parts of the logarithmic volumetric strain can be expressed, respectively, as:

$$
\theta^{e}:=-\ln \frac{v}{v_{p}} \quad \theta^{p}:=-\ln \frac{v_{p}}{v_{0}}
$$

Hence, substitution of eqns (3.16) and (3.18) leads to the following constitutive equations:

$$
\theta^{e}=k^{*} \ln \left(\frac{p}{p_{0}}\right) \quad \theta^{p}=\left(\lambda^{*}-k^{*}\right) \ln \left(\frac{p_{c}}{p_{c 0}}\right)
$$

whose rate form is:

$$
\dot{\theta}^{e}=k^{*} \frac{\dot{p}}{p} \quad \dot{\theta}^{p}=\left(\lambda^{*}-k^{*}\right) \frac{\dot{p}_{c}}{p_{c}}
$$

It can be shown that bilogarithmic relations (3.16-3.17) and their implied eqns (3.20-3.21) do not produce the unrealistic predictions previously discussed with reference to the $v-\ln p$ laws. For instance, both elastic and elasto-plastic tangent compressibilities do not depend on $v$. Furthermore, the hypothesis of solid phase incompressibility $\left(V_{s} \cong V_{s 0}\right)$ is no longer a requirement and (3.16-3.17) can be directly written in terms of the total clay volume $V$.

Bilogarithmic relations (3.16-3.17) are implemented in a number of infinitesimalstrain models of Cam-clay type (e.g. Wroth and Houlsby, 1980; Houlsby et al., 1982; AlTabbaa, 1987). However, adopting bilogarithmic equations instead of the $v-\ln p$ ones, without extending the model to finite strains is not a consistent approach, as shown below. 
Infinitesimal-strain hypothesis imply $\theta \cong E_{v}$ similarly: $E_{v}^{e} \cong \theta^{e}$ and $E_{v}^{p} \cong \theta^{p}$. Substitution of $E_{v}^{e}, E_{v}^{p}$ eqn (3.6) and of $\theta^{e}, \theta^{p}$ eqn (3.20) leads to:

$$
v \cong v_{p}-v_{0} k^{*} \ln \left(\frac{p}{p_{0}}\right) \quad v_{p}-v_{0} \cong-v_{0}\left(\lambda^{*}-k^{*}\right) \ln \left(\frac{p_{c}}{p_{c 0}}\right)
$$

which are coincident with eqns (3.1) and (3.4), respectively, setting:

$$
k=v_{0} k^{*} \quad \lambda=v_{0} \lambda^{*}
$$

Therefore, the infinitesimal-strain hypothesis implies that the relations $v-\ln p$ and $\ln v-\ln p$ are equivalent; on the contrary, it is experimentally proven that the latter better describe the mechanical behavior of clays (Butterfield, 1979).

Hence, it is not consistent to adopt bilogarithmic pressure-volume relations while continuing to consider the infinitesimal-strain hypothesis. On the contrary, the ability of the $\ln v-\ln p$ relations to reproduce the experimental behavior, calls for a finite-strain approach.

Remark III.2 Expressions (3.23) explain why $k^{*}=k / v$ and $\lambda^{*}=\lambda / v$ are often reported in the literature (e.g. Houlsby et al., 1982) as "approximated" relations between $\left(k^{*}, \lambda^{*}\right)$ and $(k, \lambda)$.

\section{A FINITE STRAIN CAM-CLAY MODEL}

In the following, the general formulation of a class of constitutive laws based on a multiplicative elasto-plastic decomposition of the deformation gradient is briefly recalled in Section IV.1. Within this framework, a finite strain Cam-clay model is formulated in Section IV.2.

\section{IV.1. Basic preliminaries}

The multiplicative theory of elasto-plasticity is based on the following decomposition formula:

$$
\mathbf{F}=\mathbf{F}^{e} \mathbf{F}^{p}
$$

where $\mathbf{F}^{e}$ and $\mathbf{F}^{p}$ are indicated as the elastic and the plastic part of the deformation gradient tensor, respectively. This decomposition follows from the notion of intermediate configuration, which is defined as the configuration obtained by unloading the current configuration. The formula (4.1) was first considered in (Lee and Liu, 1967; Lee, 1969).

As an effect of multiplicative decomposition eqn (4.1), the elastic left Cauchy-Green strain tensor $\mathbf{b}^{e}:=\mathbf{F}^{e} \mathbf{F}^{e} \mathbf{t}$ can be expressed as:

$$
\mathbf{b}^{e}=\mathbf{F} \mathbf{C}^{p-1} \mathbf{F}^{\mathbf{t}}
$$

where $\mathbf{C}^{p}:=\mathbf{F}^{p^{t}} \mathbf{F}^{p}$ is the plastic right Cauchy-Green strain tensor.

The Lie derivative of the elastic left Cauchy-Green strain tensor is defined as: 


$$
L_{v} \mathbf{b}^{e}:=\mathbf{F}\left[\frac{\partial}{\partial t}\left(\mathbf{F}^{-1} \mathbf{b}^{e} \mathbf{F}^{-\mathbf{t}}\right)\right] \mathbf{F}^{\mathbf{t}}
$$

substitution of eqn (4.2) leads to:

$$
L_{v} \mathbf{b}^{e}=\mathbf{F} \frac{\partial}{\partial t}\left(\mathbf{C}^{p^{-1}}\right) \mathbf{E}^{\mathbf{t}}
$$

Finally, the time derivative of $\mathbf{b}^{e}$ is recovered from expression (4.2) as:

$$
\dot{\mathbf{b}}^{e}=\mathbf{l b}^{e}+\mathbf{b}^{e} \mathbf{l}^{\mathbf{t}}+L_{v} \mathbf{b}^{e}
$$

where $\mathbf{l}=\dot{\mathbf{F}} \mathbf{F}^{-1}$ is the spatial velocity gradient.

The attention is now focused on the class of multiplicative elasto-plastic laws with a convex yield function defined on the current configuration (Simo, 1992).

IV.1.1. Elastic domain. The elastic domain can be defined on the current configuration as:

$$
E_{d}:=\left\{(\tau, i) \in \operatorname{sym}^{+} \times R \mid \check{f}(\tau, i) \leq 0\right\}
$$

where $\breve{f}(\boldsymbol{\tau}, i)$ is a convex yield function expressed in terms of the Kirchhoff stress $\boldsymbol{\tau}$ and of the stress-like hardening variable $i$.

It can be shown that the Principle of Objectivity restricts the choice of $\check{f}(\tau, i)$ to isotropic functions. Consistently with this restriction also the elastic response is assumed to be isotropic. Hence, the elastic strain energy function can be expressed as $\check{W}\left(\mathbf{b}^{e}\right):$ Sym $^{+} \rightarrow R$ (Chadwick, 1976).

IV.1.2. Plastic dissipation. The free energy is defined as:

$$
\psi\left(\mathbf{b}^{e}, \xi\right):=\breve{W}\left(\mathbf{b}^{e}\right)+H(\xi)
$$

where $H$ is the energy associated to the strain-like hardening variable $\xi$.

In a purely mechanical theory, the energy dissipation density is defined as the difference between the stress power and the rate of change of the free energy:

$$
D:=\boldsymbol{\tau} \cdot \mathbf{d}-\frac{\mathrm{d} \psi\left(\mathbf{b}^{e}, \xi\right)}{\mathrm{d} t}
$$

where, according to the adopted sign convention, $\mathbf{d}:=-$ sym $\mathbf{I}$ is the spatial rate of deformation. For the case herein considered, $D$ coincides with the plastic dissipation density.

The Clausius-Plank law requires the energy dissipation to be non-negative for all admissible processes: $D \geq 0$. Hence, using eqn (4.5) and following a standard argument (Coleman and Gurtin, 1967), time differentiation of the free energy function eqn (4.7) leads to:

$$
\boldsymbol{\tau}=-2 \frac{\partial \psi\left(\mathbf{b}^{e}, \xi\right)}{\partial \mathbf{b}^{e}} \mathbf{b}^{e} \quad D=\tau \cdot\left[\frac{1}{2}\left(L_{v} \mathbf{b}^{e}\right) \mathbf{b}^{\mathrm{e}^{-1}}\right]-i \dot{\xi} \geq 0
$$


where $i$ is the stress-like hardening variable conjugated to $\xi$ :

$$
i:=\frac{\partial \psi\left(\mathbf{b}^{e}, \xi\right)}{\partial \xi}
$$

Due to the specific free energy definition (4.7), (eqn (4.9) $)_{1}$ ) and (4.10) can be rewritten, respectively, as:

$$
\boldsymbol{\tau}=-2 \frac{\partial \check{W}\left(\mathbf{b}^{e}\right)}{\partial \mathbf{b}^{e}} \mathbf{b}^{e} \quad i=\frac{\partial H(\xi)}{\partial \xi}
$$

where eqn $\left(4.11_{1}\right)$ is the hyperelastic constitutive relation between the Kirchhoff stress tensor and the left Cauchy-Green strain tensor, while eqn $\left(4.11_{2}\right)$ is the hardening law.

IV.1.3. Evolutionary equations. According to the Maximum Dissipation Principle (Hill, 1951), the actual state $(\tau, i) \in E_{d}$ maximizes the dissipation function $D$. Hence, taking into account eqn $\left(4.9_{2}\right)$, the following inequality:

$$
\left[\boldsymbol{\tau}-\boldsymbol{\tau}^{*}\right] \cdot\left[\frac{1}{2}\left(L_{v} \mathbf{b}^{e}\right) \mathbf{b}^{\mathrm{e}^{-1}}\right]+\left[i-i^{*}\right][-\dot{\xi}] \geq 0
$$

holds for all admissible $\left(\boldsymbol{\tau}^{*}, i^{*}\right) \in E_{d}$. This constrained maximization problem is equivalent to the equations:

$$
\begin{aligned}
\frac{1}{2} L_{v} \mathbf{b}^{e} & =\dot{\gamma}\left[\frac{\partial \check{f}(\boldsymbol{\tau}, i)}{\partial \boldsymbol{\tau}}\right] \mathbf{b}^{e} \\
\dot{\xi} & =-\dot{\gamma}\left[\frac{\partial \check{f}(\boldsymbol{\tau}, i)}{\partial i}\right] \\
\dot{\gamma} & \geq 0 \quad \check{f}(\boldsymbol{\tau}, i) \leq 0 \quad \dot{\gamma} \check{f}(\boldsymbol{\tau}, i)=0
\end{aligned}
$$

where the constraints eqn $\left(4.13_{3}\right)$ are the plastic loading/unloading conditions expressed in the classic Kuhn-Tucker form.

IV.1.4. Reduction to principal directions. The spectral representation of the elastic left Cauchy-Green strain tensor is now considered:

$$
\mathbf{b}^{e}=\sum_{A=1}^{3}\left(\lambda_{A}^{e}\right)^{2} \quad \mathbf{n}_{A} \otimes \mathbf{n}_{A} \quad \text { where } \quad\left\|\mathbf{n}_{A}\right\|=1 \quad \text { with } \quad A=1,2,3
$$

where $\left(\mathbf{n}_{1}, \mathbf{n}_{2}, \mathbf{n}_{3}\right)$ are the principal directions and $\left(\lambda_{1}^{e}, \lambda_{2}^{e}, \lambda_{3}^{e}\right)$ are the elastic principal stretches. Hence, the vector $\boldsymbol{\rho}^{e}$ of elastic principal logarithmic stretches can be introduced as:

$$
\boldsymbol{\rho}^{e}:=\left[\begin{array}{c}
\rho_{1}^{e} \\
\rho_{2}^{e} \\
\rho_{3}^{e}
\end{array}\right] \text { where } \rho_{A}^{e}:=-\ln \lambda_{A}^{e} \quad \text { with } \quad A=1,2,3
$$


As a consequence of the isotropic elastic response, the principal directions of the Kirchhoff stress tensor and of the elastic left Cauchy-Green tensor $\mathbf{b}^{e}$ coincide; hence, the spectral decomposition of $\tau$ takes the form:

$$
\boldsymbol{\tau}=\sum_{A=1}^{3} \beta_{A} \quad \mathbf{n}_{A} \otimes \mathbf{n}_{A}
$$

Taking again into account the isotropy assumption, the elastic strain energy can be expressed as a function of the elastic principal stretches (Ogden, 1984) and, therefore, as a function of their logarithms: $\breve{W}\left(\mathbf{b}^{e}\right)=\hat{W}\left(\boldsymbol{\rho}^{e}\right)$. Hence, recalling the definition of $\boldsymbol{\rho}^{e}$ eqn (4.15) and introducing the vector of principal Kirchhoff stresses:

$$
\boldsymbol{\beta}:=\left[\begin{array}{l}
\beta_{1} \\
\beta_{2} \\
\beta_{3}
\end{array}\right]
$$

Equation $\left(4.11_{1}\right)$ implies

$$
\boldsymbol{\beta}=\frac{\partial \hat{W}\left(\boldsymbol{\rho}^{e}\right)}{\partial \boldsymbol{\rho}^{e}}
$$

The isotropic yield function can be expressed in terms of principal stresses: $\breve{f}(\tau, i)=\hat{f}(\boldsymbol{\beta}, i)$. Hence, its gradient can be spectrally represented as:

$$
\frac{\partial \check{f}(\boldsymbol{\tau}, i)}{\partial \boldsymbol{\tau}}=\sum_{A=1}^{3}\left[\frac{\partial \hat{f}(\boldsymbol{\beta}, i)}{\partial \boldsymbol{\beta}}\right]_{A} \mathbf{n}_{A} \otimes \mathbf{n}_{A}
$$

\section{IV.2. Model formulation}

A finite-strain Cam-clay model is now formulated within the multiplicative elastoplastic framework discussed so far.

IV.2.1. Kinematic preliminaries. Since the volume-preserving part of $\mathbf{F}^{e}$ is $\overline{\mathbf{F}}^{e}:=J^{e^{-1 / 3}} \mathbf{F}^{e}$, with $J^{e}:=\operatorname{det}\left[\mathbf{F}^{e}\right]$, the volumetric-deviatoric split of the elastic deformation gradient is $\mathbf{F}^{e}=J^{\mathrm{e}^{1 / 3}} \overline{\mathbf{F}}^{e}$, with $\operatorname{det}\left[\overline{\mathbf{F}}^{e}\right]=1$. Consistently with this formula, the following decomposition of vector $\boldsymbol{\rho}^{e}$ can be introduced:

$$
\boldsymbol{\rho}^{e}=\frac{1}{3} \breve{\theta}^{e} \mathbf{1}+\mathbf{e}^{e} \quad \text { with } \quad \mathbf{1}:=\left[\begin{array}{l}
1 \\
1 \\
1
\end{array}\right]
$$

where:

$$
\check{\theta}^{e}:=-\ln J^{e}=-\ln \left(\lambda_{1}^{e} \lambda_{2}^{e} \lambda_{3}^{e}\right)
$$


is the elastic logarithmic volumetric strain, while:

$$
\mathbf{e}^{e}:=\left[\begin{array}{l}
e_{1}^{e} \\
e_{2}^{e} \\
e_{3}^{e}
\end{array}\right] \text { with } e_{A}^{e}:=-\ln \left(J^{e^{-\frac{1}{3}}} \lambda_{A}^{e}\right)
$$

is the vector of elastic principal logarithmic distortions; in fact, the quantities $\bar{\lambda}_{\breve{A}}^{e 2}:=\left(J^{e^{-1 / 3}} \lambda_{A}^{e}\right)^{2}$ are the eigenvalues of the volume-preserving part of the elastic left Cauchy-Green strain tensor: $\overline{\mathbf{b}}^{e}:=\overline{\mathbf{F}}^{e} \overline{\mathbf{F}}^{\mathrm{et}}=J^{e^{-2 / 3}} \mathbf{b}^{e}$.

The multiplicative decomposition formula (4.1) implies $J=J^{e} J^{p}$, with $J^{p}:=\operatorname{det}\left[\mathbf{F}^{p}\right]$. Hence, defining the total logarithmic volumetric strain as*:

$$
\theta:=-\ln J
$$

it follows:

$$
\theta=\check{\theta}^{e}+\check{\theta}^{p}
$$

where $\check{\theta}^{p}:=-\ln J^{p}$ is the plastic logarithmic volumetric strain.6

IV.2.2. Elastic stress-strain law. The elastic strain energy density is assumed in the form:

$$
\hat{W}\left(\boldsymbol{\rho}^{e}\right):=p_{0} \check{k} \exp \left(\frac{\check{\theta^{e}}}{\check{k}}\right)+\alpha p_{0} \exp \left(\frac{\check{\theta^{e}}}{\check{k}}\right)\left\|\mathbf{e}^{e}\right\|^{2}
$$

where $\alpha$ and $\check{k}$ are positive dimensionless material constants, and, as shown in the following, $p_{0}$ is the initial pressure. The isotropic elastic potential eqn (4.25) is obtained from the one proposed by Houlsby (1985) as an extension to the finite-strain framework. According to eqn (4.18), the vector of principal Kirchhoff stresses is obtained as:

$$
\begin{aligned}
\boldsymbol{\beta} & =\frac{\partial \hat{W}}{\partial \check{\theta}^{e}} \frac{\partial \check{\theta}^{e}}{\partial \boldsymbol{\rho}^{e}}+\left(\frac{\partial \mathbf{e}^{e}}{\partial \boldsymbol{\rho}^{e}}\right) \frac{\partial \hat{W}}{\partial \mathbf{e}^{e}}=\frac{\partial \hat{W}}{\partial \check{\theta}^{e}} \mathbf{1}+\left(\mathbf{I}-\frac{1}{3} \mathbf{1} \otimes \mathbf{1}\right) \frac{\partial \hat{W}}{\partial \mathbf{e}^{e}} \\
& =p_{0} \exp \left(\frac{\check{\theta^{e}}}{\check{k}}\right)\left(\mathbf{1}+\frac{\alpha}{\check{k}}\left\|\mathbf{e}^{e}\right\|^{2}\right) \mathbf{1}+2 \alpha p_{0} \exp \left(\frac{\check{\theta^{e}}}{\check{k}}\right) \mathbf{e}^{e}
\end{aligned}
$$

The vector $\beta$ can be also written as:

$$
\boldsymbol{\beta}=\check{p} \mathbf{1}+\mathbf{t}
$$

with

$$
\check{p}=p_{0} \exp \left(\frac{\check{\theta^{e}}}{\check{k}}\right)\left(1+\frac{\alpha}{\check{k}}\left\|\mathbf{e}^{e}\right\|^{2}\right) \quad \mathbf{t}=2 \alpha p_{0} \exp \left(\frac{\check{\theta^{e}}}{\check{k}}\right) \mathbf{e}^{e}
$$

\footnotetext{
*This definition of $\theta$ coincides with that given by eqn (3.8), as shown in Section 5.1.
} 
Using the definition of $\beta$ and its decomposition eqn (4.27), is easy to verify that:

$$
\check{p}=\frac{1}{3} \operatorname{tr}(\boldsymbol{\tau}) \quad \mathbf{t}=\left[\begin{array}{l}
\bar{\beta}_{1} \\
\bar{\beta}_{2} \\
\bar{\beta}_{3}
\end{array}\right]
$$

where $\left(\bar{\beta}_{1}, \bar{\beta}_{2}, \bar{\beta}_{3}\right)$ are the eigenvalues of $\operatorname{dev}(\boldsymbol{\tau})$. Hence, $\check{p}$ is the Kirchhoff pressure, while $\mathbf{t}$ is the vector of principal deviatoric Kirchhoff stresses. It can be observed that $\|\mathbf{t}\|=\|\operatorname{dev}(\boldsymbol{\tau})\|$.

Furthermore, indicating with $\sigma$ the Cauchy stress and recalling that $\tau:=J \sigma$, the following relations between the invariants of $\boldsymbol{\tau}$ and of $\boldsymbol{\sigma}$ are recovered:

$$
\check{p}=J p \quad\|\mathbf{t}\|=J\|\operatorname{dev}(\sigma)\|
$$

Remark IV.1: Setting $\breve{\theta^{e}}=0$ and $\mathbf{e}^{e}=\mathbf{0}$ in the constitutive eqn $\left(4.28_{1}\right), \check{p}=p_{0}$ is obtained; hence, $p_{0}$ is the initial pressure. Obviously, being $J=1$ in the reference configuration, $\left(4.30_{1}\right)$ implies that the Kirchhoff and the Cauchy initial pressures coincide.

Remark IV.2: It is interesting to observe the coupling between the volumetric and the deviatoric parts of the non-linear elastic constitutive law. To evaluate the effects of relations (4.28) on the predicted shearing and isotropic compression response, the following equations are considered:

$$
\begin{gathered}
\|\mathbf{t}\|=\frac{2 \alpha \check{k}\left\|\mathbf{e}^{e}\right\|}{\check{k}+\alpha\left\|\mathbf{e}^{e}\right\|^{2}} \check{p} \\
\check{p}=p_{0} \exp \left(\frac{\check{\theta^{e}}}{\check{k}}\right)\left[1+\frac{\|\mathbf{t}\|^{2}}{4 \alpha \check{k}_{p 0^{2}}^{2}} \exp \left(-2 \frac{\check{\theta^{e}}}{\check{k}}\right)\right]
\end{gathered}
$$

obtained combining eqn (4.281) with eqn (4.282). These relations are plotted in Fig. 5 setting $\check{k}=0.05, \alpha=100$ and $p_{0}=100 \mathrm{kPa}$; this choice falls within the range usually considered for clays (see Section V.3).

(a)

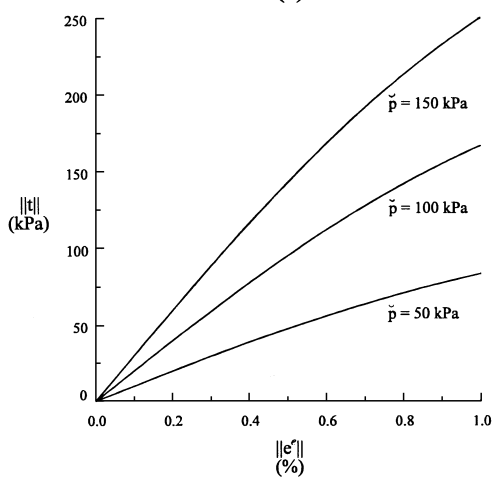

(b)

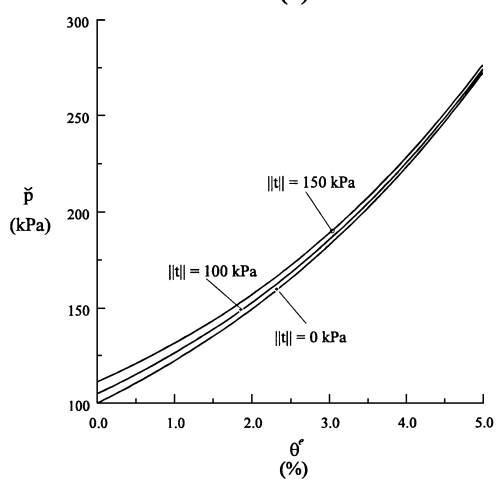

Fig. 5. Elastic stress-strain law. Influence of the pressure $\breve{p}$ on the shear tangent stiffness (a) and of the deviatoric stress $\|\mathbf{t}\|$ on the bulk tangent stiffness (b). 
Graphs of eqn (4.31) relative to constant pressure paths $(\check{p}=50, \check{p}=100$ and $\check{p}=150 \mathrm{kPa}$ ) are plotted in a $\|\mathbf{t}\|-\left\|\mathbf{e}^{e}\right\|$ diagram (Fig. 5(a)). It is apparent that the shear tangent stiffness increases with $\check{p}$. Hence, differently from other formulations (see Remark 2.1), this realistic response is obtained using a conservative stress-strain relation.

Graphs of eqn (4.32) relative to constant deviatoric stress paths $(\|\mathbf{t}\|=0,\|\mathbf{t}\|=100$ and $\|\mathbf{t}\|=150 \mathrm{kPa}$ ) are plotted in a $\check{p}-\check{\theta}^{e}$ diagram (Fig. 5(b)). These curves point out a slight influence of $\|\mathbf{t}\|$ on the bulk tangent stiffness. In fact, increasing $\check{\theta}^{e}$, the second addendum of eqn (4.32) decreases and the curves tend to overlap.

A detailed experimental study of the predictive ability of elastic constitutive law by Houlsby (1985) can be found in (Borja et al., 1997).

IV.2.3. Yield criterion. The following convex and isotropic yield function, defined on the current configuration, is considered:

$$
\hat{f}\left(\beta, \check{p}_{c}\right)=\frac{\check{q}^{2}}{M^{2}}+\check{p}\left(\check{p}-\check{p}_{c}\right)=0
$$

Equation (4.33) is the Modified Cam-clay criterion eqn (2.5) expressed in terms of Kirchhoff stress invariants. In particular, $\check{q}:=\sqrt{3 / 2}\|\operatorname{dev}(\boldsymbol{\tau})\|=\sqrt{3 / 2}\|\mathbf{t}\|=J q$ and the preconsolidation pressure $\check{p}_{c}$ is the stress-like hardening variable $\left(i=\check{p}_{c}\right)$. It can be observed that the slope $M$ of the critical state line in the $p-q$ plane is coincident with the slope of the same line in the $\breve{p}-\check{q}$ plane; in fact, and $q / p=\breve{q} / \check{p}$.

IV.2.4. Flow rule Taking into account the spectral decomposition eqn (4.19), the evolutionary equation $\left(4.13_{1}\right)$ can be rewritten as:

$$
\frac{1}{2} L_{v} \mathbf{b}_{e}=\dot{\gamma}\left[\sum_{A=1}^{3}\left(\frac{\partial \hat{f}(\boldsymbol{\beta}, \check{p})_{c}}{\partial \boldsymbol{\beta}}\right)_{A} \mathbf{n}_{A} \otimes \mathbf{n}_{A}\right] \mathbf{b}^{e}
$$

Recalling expression (4.27), the derivative of the yield function with respect to $\beta$ can be computed as:

$$
\frac{\partial \hat{f}\left(\boldsymbol{\beta}, \check{p}_{c}\right)}{\partial \boldsymbol{\beta}}=\frac{\partial \hat{f}}{\partial \check{p}} \frac{\partial \check{p}}{\partial \boldsymbol{\beta}}+\left(\frac{\partial \mathbf{t}}{\partial \boldsymbol{\beta}}\right) \frac{\mathbf{t}}{\partial \hat{f}} \frac{1}{\partial \mathbf{t}}=\frac{\partial \hat{f}}{3} \frac{1}{\partial \check{p}}+\left(\mathbf{I}-\frac{1}{3} \mathbf{1} \otimes \mathbf{1}\right) \frac{\partial \hat{f}}{\partial \mathbf{t}}=\left[\frac{1}{3}\left(2 \check{p}-\check{p}_{c}\right) \mathbf{1}+\frac{3}{M^{2}} \mathbf{t}\right]
$$

Substituting in eqn (4.34), the following flow rule is obtained:

$$
\frac{1}{2} L_{v} \mathbf{b}^{e}=\dot{\gamma}\left[\sum_{A=1}^{3}\left(\frac{1}{3}\left(2 \check{p}-\check{p}_{c}\right)+\frac{3}{M^{2}} t_{A}\right) \mathbf{n}_{A} \otimes \mathbf{n}_{A}\right] \mathbf{b}^{e}
$$

IV.2.5. Hardening law. The plastic volumetric logarithmic strain is chosen as hardening variable $\left(\xi=\check{\theta}^{p}\right)$. The corresponding energy is assumed as: 


$$
H\left(\check{\theta}^{p}\right)=\check{p}_{c 0}(\check{\lambda}-\check{k}) \exp \left(\frac{1}{\check{\lambda}-\check{k}} \check{\theta}^{p}\right)
$$

where $\check{\lambda}$ is a dimensionless material constant and $\check{p}_{c 0}$ fixes the initial size of the yield curve. Recalling eqn $\left(4.11_{2}\right)$, the following hardening law is obtained:

$$
\check{p}_{c}=\frac{\partial H\left(\check{\theta}^{p}\right)}{\partial \check{\theta}_{p}}=\check{p}_{c 0} \exp \left(\frac{1}{\check{\lambda}_{\bar{\lambda}} \check{\theta}^{p}}\right)
$$

Remark IV.1: It can be shown that the evolution of $\check{\theta}^{p}$ prescribed by the associative flow rule eqn (4.36) is different from that one implied by eqn $\left(4.13_{2}\right)$, i.e.:

$$
\dot{\check{\theta}}^{p} \neq-\dot{\gamma}\left[\frac{\partial \hat{f}\left(\boldsymbol{\beta}, p_{c}\right)}{\partial p_{c}}\right]
$$

hence, the adopted hardening law is "non-associative".

\section{ANALYSIS OF THE MODEL}

The major implications of the finite-strain Cam-clay model herein presented are now discussed in detail.

\section{V.1. Isotropic compression $\check{p}-v$ relations}

As recalled in Section III.1, $J:=\operatorname{det}[\mathbf{F}(\mathbf{X})]$ measures the current volume for unit volume in the reference configuration; hence, the specific volume definition (2.1) implies:

$$
J=\frac{v V_{s}}{v_{0} V_{s 0}}
$$

where $V_{s 0}$ and $V_{s}$, are the initial and the current volume of the solid phase, respectively, while $v_{0}$ is the initial specific volume. Assuming that the compressibility of the solid phase is negligible ${ }^{\dagger}$, i.e. $V_{s}=V_{s 0}$, then:

$$
J=\frac{v}{v_{0}}
$$

Therefore, the definition of the logarithmic volumetric strain eqn (4.23) and the decomposition eqn (4.24) imply, respectively:

$$
\theta=-\ln \left(\frac{v}{v_{0}}\right)
$$

\footnotetext{
†This hypothesis is not a requirement for the formulation of the present model (see Section III.2). However, the solid phase contribution to volumetric strains of clays is normally negligible. For this reason, $v$ is the most frequently adopted volumetric measure, especially in the experimental context. Accordingly, instead of the total volume, the specific volume is still considered in this section.
} 


$$
\check{\theta}^{e}=-\ln \left(\frac{v}{\check{v}_{p}}\right) \quad \check{\theta}^{p}=-\ln \left(\frac{\check{v}_{p}}{v_{0}}\right)
$$

where, as shown in the following, the quantity $\check{v}_{p}$ has the same physical meaning of the quantity $v_{p}$ introduced in Section III.

The elastic isotropic compression constitutive relation is obtained setting $\mathbf{e}^{e}=\mathbf{0}$ in eqn (4.281):

$$
\check{p}=p_{0} \exp \left(\frac{\check{\theta^{e}}}{\check{k}}\right)
$$

Substitution of eqn $\left(5.4_{1}\right)$ gives the following pressure-volume relation for overconsolidated clays:

$$
\ln \left(\frac{v}{\check{v}_{p}}\right)=-\check{k} \ln \left(\frac{\check{p}}{p_{0}}\right)
$$

It can be observed that $\breve{v}_{p}$ is the volume obtained unloading the current configuration to the initial pressure value. Hence, $\check{v}_{p}$ is the specific volume in the intermediate configuration.

To obtain the elasto-plastic isotropic compression constitutive relation, eqn $\left(5.4_{2}\right)$ is substituted into the hardening law eqn (4.38):

$$
\ln \left(\frac{\check{v}_{p}}{v_{0}}\right)+(\check{\lambda}-\check{k}) \ln \left(\frac{\check{p}_{c}}{\check{p}_{c 0}}\right)=0
$$

Furthermore, indicating with $v_{c 0}$ and $v_{c}$ the specific volumes corresponding to $\check{p}_{c 0}$ and $\check{p}_{c}$, respectively, it is easy to verify that (Fig. 6):

$$
\ln \left(\frac{v_{c}}{v_{c 0}}\right)=\ln \left(\frac{\check{v}_{p}}{v_{0}}\right)-\check{k} \ln \left(\frac{\check{p}_{c}}{\check{p}_{c 0}}\right)
$$

Recalling eqn (5.7), the pressure-volume relation for normally consolidated clays is obtained:

$$
\ln \left(\frac{v_{c}}{v_{c 0}}\right)=-\check{\lambda} \ln \left(\frac{\check{p}_{c}}{\check{p}_{c 0}}\right)
$$

Hence, for the case of isotropic compression, the considered Cam-clay model reduces to $v-\ln p$ linear relations. As pointed out in Section III, these bilogarithmic laws do not lead to the unrealistic behaviors predicted by $v-\ln p$ linear relations, assumed in the original and in the Modified Cam-clay.

V.1.1. Material classification problem. The classification of the elastic material described by the energy function (4.25) is now considered.

The bilogarithmic relation (3.16) between the Cauchy pressure $p$ and the specific volume $v$ can be written in the form: $p v^{1 / k^{*}}=p_{0} v_{p}^{1 / k^{*}}$, which characterizes adiabatic transformations of gases. This fact is not just a formal analogy, as observed by Butterfield (1979), but the solution of the relative classification problem, as shown in the following. 


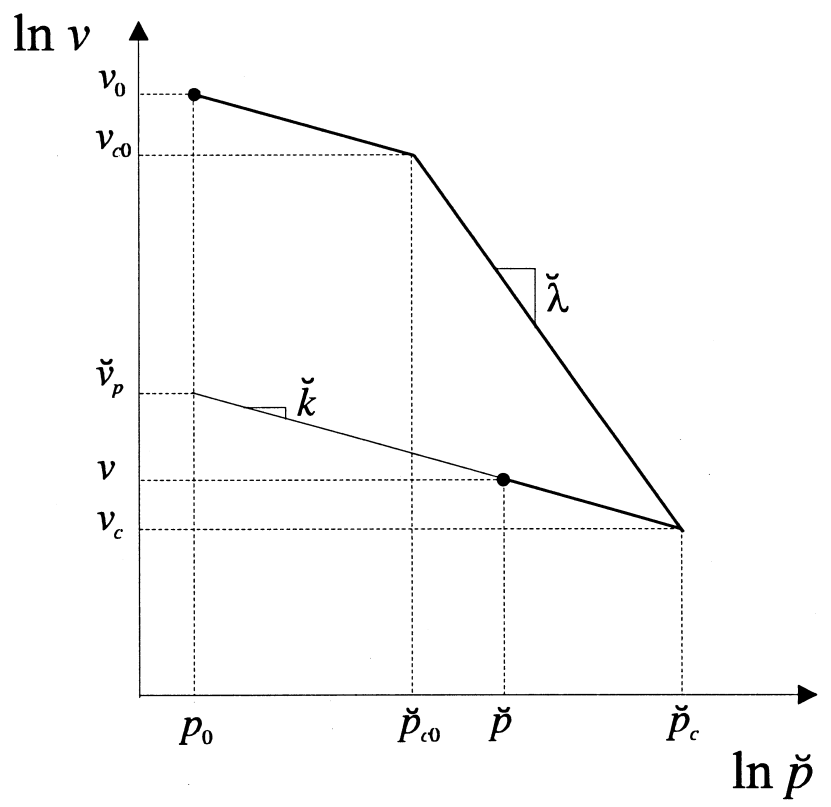

Fig. 6. Bilogarithmic relations between Kirchhoff pressure $(\breve{p})$ and specific volume $(v)$.

Recalling the volumetric-deviatoric decomposition of the deformation gradient $\mathbf{F}^{e}=J^{1 / 3} \overline{\mathbf{F}}^{e}$, with $\operatorname{det}\left[\overline{\mathbf{F}}^{e}\right]=1$, the elastic strain energy can be expressed as $W\left(\mathbf{F}^{e}\right)=$ $\hat{w}_{v}\left(J^{e}, \overline{\mathbf{F}}^{e}\right)$. Then, following (Podio-Guidugli and Vergara Caffarelli, 1991), the volumetric sub-energy can be defined as:

$$
w_{v}\left(J^{e}\right):=\inf \left[\hat{w}_{v}\left(J^{e}, \overline{\mathbf{F}}^{e}\right)\right]_{\operatorname{det}\left[\overline{\mathbf{F}}^{e}\right]=1}
$$

A material whose elastic strain energy is given as $W\left(\mathbf{F}^{e}\right)=h\left(J^{e}\right)$ is classified as a fluid (e.g. see Marsden and Hughes, 1983); among fluids, the distinction between liquids and gases can be done analysing the fluid behavior under extreme deformations.

From the strain energy function eqn (4.25), setting $\overline{\mathbf{F}}^{e}=\mathbf{I}$, i.e. $\mathbf{e}^{e}=\mathbf{0}$, the corresponding volumetric sub-energy is obtained as:

$$
w_{v}\left(J^{e}\right)=p_{0} \check{k} J^{e^{-1 / \check{k}}}
$$

and for the case of extreme deformations it gives:

$$
\begin{aligned}
& J^{e} \rightarrow 0 \quad\left(\theta^{e} \rightarrow \infty\right) \quad \Rightarrow \quad w_{v}\left(J^{e}\right) \rightarrow \infty \\
& J^{e} \rightarrow \infty \quad\left(\theta^{e} \rightarrow-\infty\right) \quad \Rightarrow \quad w_{v}\left(J^{e}\right) \rightarrow 0
\end{aligned}
$$

Noting that the strain energy goes to zero when the material is indefinitely expanded, the condition to classify a fluid as a gas is verified. Hence, the constitutive elastic behavior in isotropic compression prescribed by eqn (4.25) and, in general, by $\ln v-\ln p$ linear relations, is to be classified as gas-like. 
It can be also observed that $w_{v}(1)=p_{0} \check{k}>0$. Hence, for nil volumetric strain $\left(J^{e}=1, \theta^{e}=0\right)$, the corresponding energy is not nil.

V.1.2. Comparison between "Cauchy" and "Kirchhoff" bilogarithmic relations. The comparison between the bilogarithmic relations in terms of Kirchhoff pressures eqn (5.6), eqn (5.9) and the analogous ones in terms of Cauchy pressures eqns (3.16-3.17) is now considered.

For instance, consider to isotropically compress a normally consolidated sample $\left(p_{0}=p_{c 0}\right)$. According to relation eqn (3.17), its response is (Fig. 7):

$$
\ln \left(\frac{v}{v_{0}}\right)=-\lambda^{*} \ln \left(\frac{p}{p_{0}}\right)
$$

while, in terms of Kirchhoff pressures, relation (5.9) gives:

$$
\ln \left(\frac{v}{v_{0}}\right)=-\check{\lambda} \ln \left(\frac{\check{p}}{p_{0}}\right)
$$

Substitution of $\breve{p}=J p$ in the last leads to:

$$
\ln \left(\frac{v}{v_{0}}\right)=-\check{\lambda} \ln \left(\frac{J p}{p_{0}}\right)=-\check{\lambda} \ln \left(\frac{p}{p_{0}}\right)-\check{\lambda} \ln J
$$

hence, recalling eqn (5.2), it follows:

$$
\ln \left(\frac{v}{v_{0}}\right)=-\frac{\check{\lambda}}{1+\check{\lambda}} \ln \left(\frac{p}{p_{0}}\right)
$$

The comparison of eqn (5.16) with eqn (5.13) returns:

$$
\lambda^{*}=\frac{\check{\lambda}}{1+\check{\lambda}}
$$

In the same way, if the isotropic compression of an overconsolidated sample $\left(p_{0}<p_{c 0}\right)$ is considered, it can be shown that:

$$
k^{*}=\frac{\check{k}}{1+\check{k}}
$$

Accordingly, the model parameters $\check{k}$ and $\check{\lambda}$ can be recovered from the constants $k^{*}$ and $\lambda^{*}$ as:

$$
\check{k}=\frac{k^{*}}{1-k^{*}} \quad \check{\lambda}=\frac{\lambda^{*}}{1-\lambda^{*}}
$$

It can be observed that for physically possible values of $k^{*}$ and $\lambda^{*}$, i.e. $\left.k^{*}, \lambda^{*} \in\right] 0 ; 1[$, relations (5.19) give $\check{k}>k^{*}$ and $\check{\lambda}>\lambda^{*}$ (Fig. 7). 


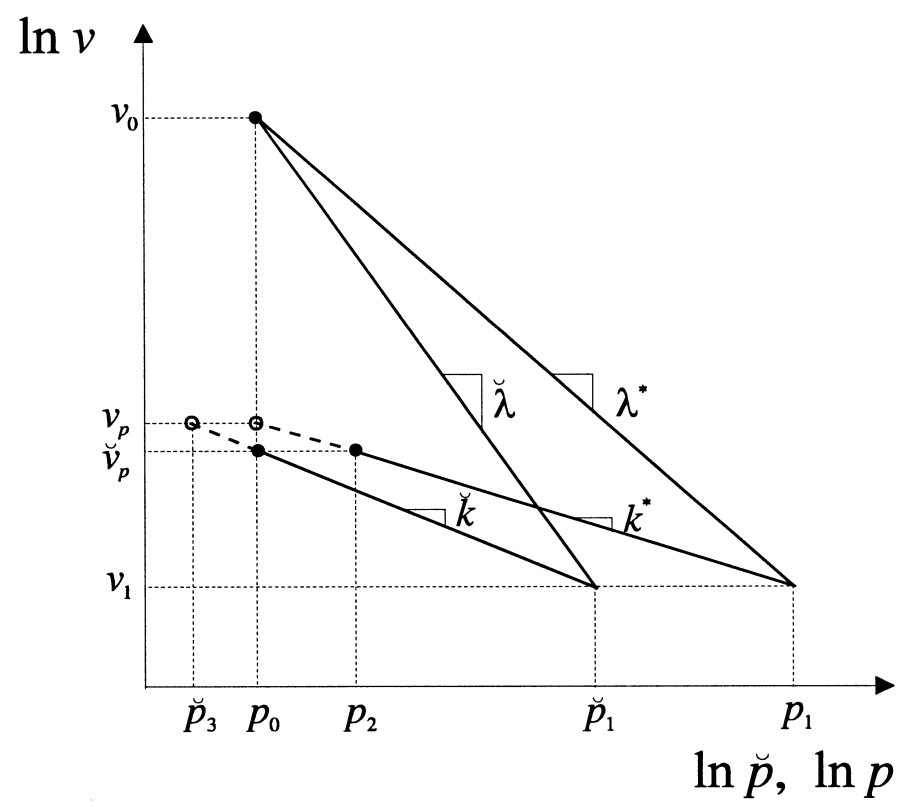

Fig. 7. Comparison between the bilogarithmic relations expressed in terms of Kirchhoff $(\breve{p})$ and Cauchy $(p)$ pressures.

Starting from the comparison between "Cauchy" and "Kirchhoff" pressure-volume relations, some considerations on the elasto-plastic decomposition of the volumetric strain are now carried out. In particular, the assumption of a non stress-free reference configuration results in two different elasto-plastic decompositions of the volumetric strain, depending if the "Cauchy" or the "Kirchhoff" pressure-volume relations are considered.

Let a normally consolidated sample $\left(p_{0}=\check{p}_{c 0}\right)$ be isotropically compressed to the value $\check{p}_{1}$ of Kirchhoff pressure; then, it is unloaded to the initial pressure $\check{p}_{2}=p_{0}$. In Fig. 7, the $\ln \check{p}-\ln v$ curve relative to this loading-unloading path is plotted together with the corresponding one in terms of Cauchy pressure. It can be observed that when the Kirchhoff pressure is reduced to the initial value $p_{0}$, the Cauchy pressure does not recover the same value; in fact:

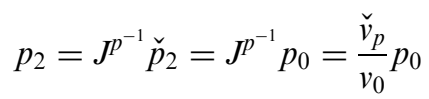

Hence, the Cauchy pressure recovers its initial value $p_{0}$ only if the sample is further unloaded; in particular, as obtained from eqn (5.6), the following Kirchhoff pressure must be reached:

$$
\check{p}_{3}=p_{0}\left(\frac{\check{v}_{p}}{v_{p}}\right)^{1 / \check{k}}
$$

Hence:

$$
\check{v}_{p} \neq v_{p}
$$


i.e. with reference to the volume measures, the "Kirchhoff" intermediate configuration does not coincide with the "Cauchy" one. This result follows from the assumption of a non-nil initial pressure $p_{0}$, which is a requirement for every constitutive law characterized by the "gas-like" solution eqn $\left(5.12_{2}\right)$.

Recalling now definitions (3.19) and (5.4), from inequality (5.22) it follows that:

$$
\theta^{e} \neq \check{\theta}^{e} \quad \theta^{p} \neq \check{\theta}^{p}
$$

Hence, the elasto-plastic decomposition of the volumetric strain induced by constitutive laws in terms of Kirchhoff pressures (eqns (5.6 and 5.9)) does not coincide, in general, with the elasto-plastic decomposition induced by constitutive laws in terms of Cauchy pressures (eqns (3.16 and 3.17)). Clearly, the total strains coincide and in particular their volumetric components, i.e. $\theta=\check{\theta}$.

To obtain the relation between the two specific volumes $\check{v}_{p}$ and $v_{p}$, the pressure $\check{p}_{3}=J_{3} p_{0}$ is substituted in eqn (5.21) and, being $J_{3}=v_{p} / v_{0}$ (Fig. 7), it follows:

$$
\check{v}_{p}=\left(\frac{v_{p}}{v_{0}}\right)^{\check{k}} v_{p}
$$

Hence, the difference between $\check{v}_{p}$ and $v_{p}$ increases with the plastic volumetric strain.

From eqn (5.24), recalling eqn $\left(3.19_{2}\right)$ and $\left(5.4_{2}\right)$, a relation between the two plastic volumetric strain measures is obtained:

$$
\theta^{p}=\frac{1}{1+\check{k}} \check{\theta}^{p}
$$

as well as a relation between the two elastic volumetric strain measures:

$$
\theta^{e}=\theta-\theta^{p}=\check{\theta}^{e}+\check{\theta}^{p}-\theta^{p}=\check{\theta}^{e}+\check{\theta}^{p}-\frac{1}{1+\check{k}} \check{\theta}^{p}=\check{\theta}^{e}+\frac{\check{k}}{1+\check{k}} \check{\theta}^{p}
$$

In the following, only the decomposition $\theta=\check{\theta}^{e}+\breve{\theta}^{p}$ is considered; hence, to simplify the notation, the symbol " " " in $\check{\theta}^{e}$ and $\breve{\theta}^{p}$ is dropped.

Remark V.1: Considerations on elasto-plastic decomposition similar to those reported above can be in general extended to any multiplicative constitutive law formulated in terms of Kirchhoff stress tensor. In fact, in these models, similar results are obtained every time a reference configuration with a non-nil stress state is considered.

\section{V.2. Rate form of the elastic law}

The second order tensor which relates the increments $\dot{\beta}$ of the principal stresses to the increments $\dot{\boldsymbol{\rho}}^{e}$ of the elastic logarithmic stretches can be computed as:

$$
\mathbf{a}^{e}:=\frac{\partial \boldsymbol{\beta}}{\partial \boldsymbol{\rho}^{e}}=\mathbf{1} \otimes \frac{\partial \check{p}}{\partial \boldsymbol{\rho}^{e}}+\frac{\partial \mathbf{t}}{\partial \boldsymbol{\rho}^{e}}
$$


where

$$
\frac{\partial \check{p}}{\partial \boldsymbol{\rho}^{e}}=\frac{\partial \check{p}}{\partial \theta^{e}} \frac{\partial \theta^{e}}{\partial \boldsymbol{\rho}^{e}}+\left(\frac{\partial \mathbf{e}^{e}}{\partial \boldsymbol{\rho}^{e}}\right)^{\mathbf{t}} \frac{\partial \check{p}}{\partial \mathbf{e}^{e}} \quad \frac{\partial \mathbf{t}}{\partial \boldsymbol{\rho}^{e}}=\frac{\partial \mathbf{t}}{\partial \theta^{e}} \otimes \frac{\partial \theta^{e}}{\partial \boldsymbol{\rho}^{e}}+\frac{\partial \mathbf{t}}{\partial \mathbf{e}^{e}} \frac{\partial \mathbf{e}^{e}}{\partial \boldsymbol{\rho}^{e}}
$$

with:

$$
\frac{\partial \theta^{e}}{\partial \boldsymbol{\rho}^{e}}=\mathbf{1} \quad \frac{\partial \mathbf{e}^{e}}{\partial \boldsymbol{\rho}^{e}}=\mathbf{I}-\frac{1}{3} \mathbf{1} \otimes \mathbf{1}
$$

Hence, differentiation of the elastic stress-strain relations (4.28) is required:

$$
\frac{\partial \check{p}}{\partial \theta^{e}}=\frac{\check{p}}{\check{k}} \quad \frac{\partial \check{p}}{\partial \mathbf{e}^{e}}=\frac{\mathbf{t}}{\check{k}} \quad \frac{\partial \mathbf{t}}{\partial \theta^{e}}=\frac{\mathbf{t}}{\check{k}} \quad \frac{\partial \mathbf{t}}{\partial \mathbf{e}^{e}}=2 \alpha g \mathbf{I}
$$

where: $g=p_{0} \exp \left(\theta^{e} / \check{k}\right)$. Relations (5.30) are substituted in eqn (5.28):

$$
\frac{\partial \check{p}}{\partial \boldsymbol{\rho}^{e}}=\frac{1}{\check{k}}(\check{p} \mathbf{1}+\mathbf{t}) \quad \frac{\partial \mathbf{t}}{\partial \boldsymbol{\rho}^{e}}=\frac{1}{\check{k}} \mathbf{t} \otimes \mathbf{1}+2 \alpha g\left(\mathbf{I}-\frac{1}{3} \mathbf{1} \otimes \mathbf{1}\right)
$$

and the form of the elastic tangent tensor can be recovered:

$$
\mathbf{a}^{e}=\frac{\check{p}}{\breve{k}} \mathbf{1} \otimes \mathbf{1}+2 \alpha g\left(\mathbf{I}-\frac{1}{3} \mathbf{1} \otimes \mathbf{1}\right)+\frac{1}{\check{k}}(\mathbf{1} \otimes \mathbf{t}+\mathbf{t} \otimes \mathbf{1})
$$

Remark V.2: Due to the volumetric-deviatoric coupling, expression (5.32) differs from the typical Lamé formula for the presence of the third addendum.

Remark V.3: The elastic tangent tensor $\mathbf{a}^{e}$ eqn (5.32) is not positively defined; in fact:

$$
\exists \boldsymbol{\rho}^{e} \mid \mathbf{a}^{e} \dot{\boldsymbol{\rho}}^{e} \cdot \dot{\boldsymbol{\rho}}^{e} \leq 0
$$

It is possible to verify that $\operatorname{det}\left[\mathbf{a}^{e}\right] \leq 0$ is satisfied for:

$$
\left\|\mathbf{e}^{e}\right\| \geq\left(\frac{\check{k}}{\alpha}\right)^{\frac{1}{2}}
$$

To investigate the consequence of the non-positiveness of $\mathbf{a}^{e}$, the $\|\mathbf{t}\|-\left\|\mathbf{e}^{e}\right\|$ relation (4.31) is plotted in Fig. 8 for $\check{k}=0.05, \alpha=100$ and constant pressure $\check{p}=100 \mathrm{kPa}$. It can be observed that the tangent to the stress-strain curve changes sign for $\left\|\mathbf{e}^{e}\right\|=(\check{k} / \alpha)^{1 / 2} \cong 0.022$. For a soil, this behavior is unrealistic within the elastic range. Hence, to investigate the model applicability limits, condition (5.34) is expressed only in terms of stresses, substituting the limit condition $\left\|\mathbf{e}^{e}\right\|=(\breve{k} / \alpha)^{1 / 2}$ in eqn (4.31):

$$
\|\mathbf{t}\|=(\alpha \check{k})^{\frac{1}{2}} \check{p}
$$

If condition (5.35) is verified, a positive strain increment produces a negative stress increment. Hence, eqn (5.35) gives the maximum attainable deviatoric stress, which can be rewritten in terms of $\check{q}$ as: 


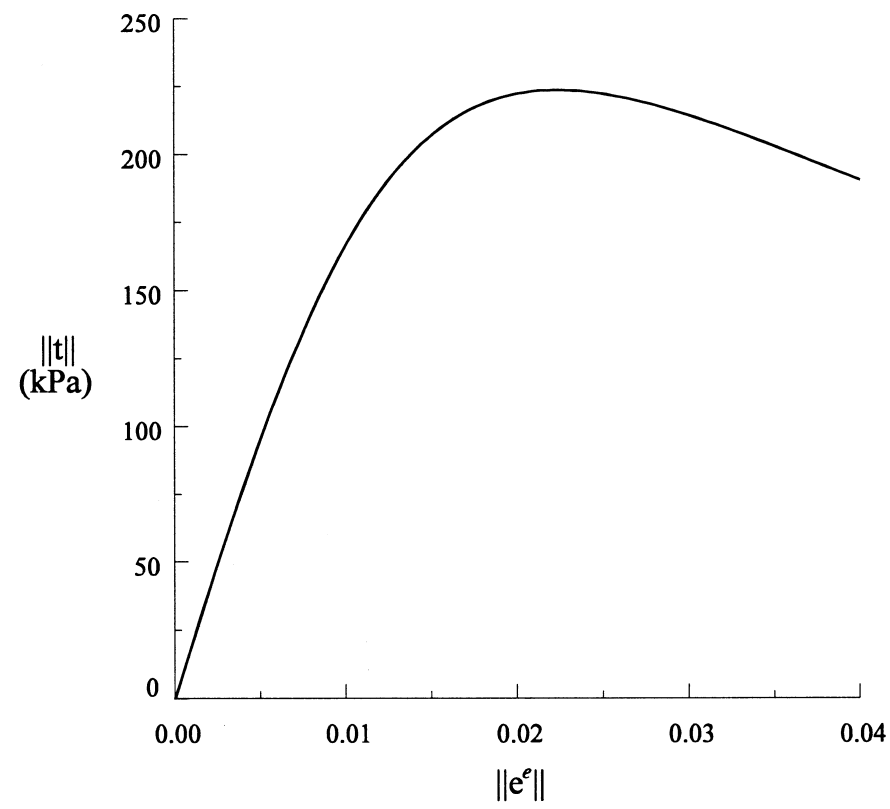

Fig. 8. Elastic stress-strain law. Sign change of the tangent shear modulus.

$$
\check{q}=\left(\frac{3 \alpha \breve{k}}{2}\right)^{\frac{1}{2}} \check{p}
$$

Such a maximum was originally observed by Houlsby (1985).

Considering parameters which normally characterize soft clays, the straight line given by the previous equation lies above the critical state line; this "super-critical" region is of real interest just for the constitutive modelling of stiff clays. As example, in Fig. 9, the graph of eqn (5.36) is plotted on the $p-\check{q}$ plane together with the CSL and the yield curve eqn (4.33) for the case $\breve{k}=0.05, \alpha=100$ and $M=0.9$.

\section{V.3. Model parameters}

The finite-strain Cam-clay model discussed in this paper is based on four parameters: $\alpha, \breve{k}, \breve{\lambda}$ and $M$. Hence, despite the introduction of $\alpha$, the parameter number is not increased with respect to the Cam-clay and Modified Cam-clay formulations (see Section II). In fact, due to the replacement of $v-\ln p$ with $\ln v-\ln p$ linear relations, the initial specific volume $v_{0}$ is no more a model constant.

The physical meaning of the four model parameters is discussed in the following.

V.3.1. Elastic parameter $\alpha$. In a clay the solid phase and the interstitial fluid compressibility can be usually neglected. Accordingly, for saturated clays in undrained conditions the volumetric strains are nil $(\theta=0)$ and the elastic constitutive eqn (4.28) can be rewritten as: 


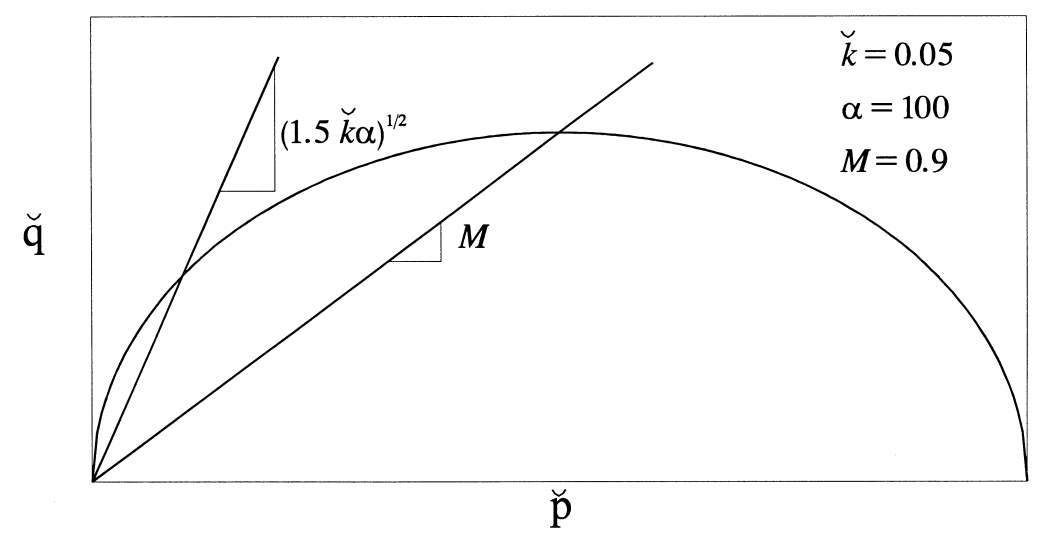

Fig. 9. Elastic stress-strain law. Line of maximum attainable deviatoric stress.

$$
\check{p}=p_{0}\left(1+\frac{\alpha}{\check{k}}\left\|\mathbf{e}^{e}\right\|^{2}\right) \quad\|\mathbf{t}\|=2 \alpha p_{0}\left\|\mathbf{e}^{e}\right\|
$$

Hence, the undrained elastic tangent shear modulus of a clay initially consolidated up to the pressure $p_{0}$ is:

$$
\mu_{u}:=\frac{1}{2} \frac{\partial\|\mathbf{t}\|}{\partial\left\|\mathbf{e}^{e}\right\|}=\alpha p_{0}
$$

and the parameter $\alpha$ can be obtained as:

$$
\alpha=\frac{\mu_{u}}{p_{0}}
$$

In undrained conditions, it is $J=1$ and $q=\check{q}=\sqrt{3 / 2}\|\mathbf{t}\|$; furthermore, if the strain measure $\check{\varepsilon}_{q}^{e}:=\sqrt{2 / 3}\left\|\mathbf{e}^{e}\right\|$ conjugated to $\breve{q}$ is introduced, it follows that:

$$
\frac{1}{3} \frac{\partial q}{\partial \check{\varepsilon}_{q}^{e}}=\frac{1}{2} \frac{\partial\|\mathbf{t}\|}{\partial\left\|\mathbf{e}^{e}\right\|}=: \mu_{u}
$$

Accordingly, the elastic parameter $\alpha$ can be obtained from a conventional undrained triaxial test. If the test is performed on a normally consolidated sample, an unloading path is required. As shown in Fig. 10, $\mu_{u}$ can be evaluated from the unloading phase results; then, using eqn (5.38), $\alpha$ can be computed.

V.3.2. Swelling parameter $\check{k}$ and virgin compression parameter $\check{\lambda}$. The two model parameters $\check{k}$ and $\lambda$ can be obtained from an isotropic compression test. If the test is performed on a normally consolidated sample, an unloading stress path is required. During such a test, Cauchy pressures are measured; therefore, relations (5.19) can be used to compute $(\breve{k}, \grave{\lambda})$ starting from the parameters $\left(k^{*}, \lambda^{*}\right)$ used in "Cauchy" relations (3.16) and (3.17). 


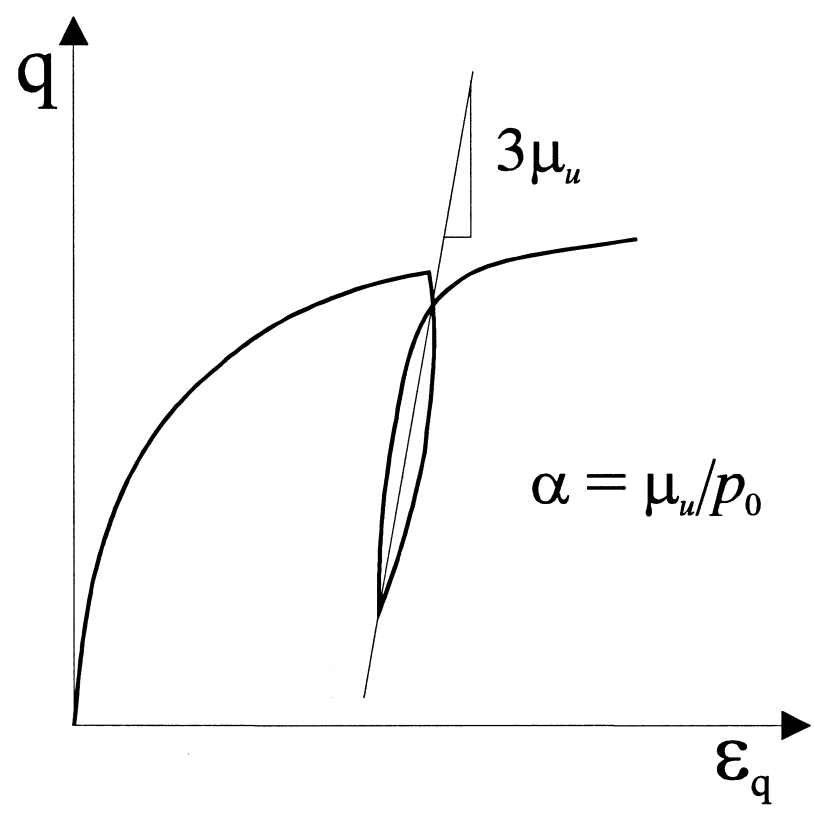

Fig. 10. Evaluation of the elastic parameter $\alpha$ from undrained triaxial test results.

V.3.3. Slope of the critical state line $M$. The parameter $M$ coincides with the one used in the infinitesimal-strain formulation of the Cam-clay model (see Section II). To obtain this parameter, a conventional drained triaxial test is required. Evaluations of $M$ for various clays are widely reported in the literature (e.g. Schofield and Wroth, 1968).

\section{V.4. Initial conditions}

The model requires the assignment of the initial values for the pressure $\left(p_{0}\right)$ and the preconsolidation pressure $\left(\check{p}_{c 0}\right)$. If the material is overconsolidated $\left(p_{0}<\check{p}_{c 0}\right)$, the Kirchhoff initial preconsolidation pressure $\check{p}_{c 0}$ can be obtained from the corresponding value in terms of Cauchy stress $p_{c 0}$ through the relation $\check{p}_{c 0}=J_{c 0} p_{c 0}$, where the quantity $J_{c 0}=v_{c 0} / v_{0}$ can be recovered, as shown in Fig. 11, using the eqn (3.16):

$$
\ln \left(\frac{v_{c 0}}{v_{0}}\right)=-k^{*} \ln \left(\frac{p_{c 0}}{p_{0}}\right)
$$

Hence, the required relation is:

$$
\check{p}_{c 0}=\left(\frac{p_{0}}{p_{c 0}}\right)^{k^{*}} p_{c 0}
$$

Although the initial specific volume is not a model constant, assignment of $v_{0}$ is required to represent the predicted response in terms of pressure-volume curves. Usually, the specific volume $v_{c 1}$ of a sample normally consolidated up to a unit pressure is recovered from 
an isotropic compression test; hence, it can be useful to derive the relation between $v_{0}$ and $v_{c 1}$. Using equations $\$$ (3.16) and (3.17) (Fig. 12):

$$
\ln \left(\frac{v_{c 0}}{v_{0}}\right)=-k^{*} \ln \left(\frac{p_{c 0}}{p_{0}}\right) \quad \ln \left(\frac{v_{c 1}}{v_{c 0}}\right)=-\lambda^{*} \ln \left(\frac{p_{c 1}}{p_{c 0}}\right)
$$

with $p_{c 1}=1$ (e.g. $\left.\mathrm{kPa}\right)$, the following relation is obtained:

$$
v_{0}=\left(\frac{p_{c 0}}{p_{0}}\right)^{k^{*}} p_{c 0}^{-\lambda^{*}} v_{c 1}
$$

\section{COMPARISON WITH EXPERIMENTAL RESULTS}

The model ability to reproduce experimental data available in the literature is now investigated. In particular, an isotropic compression and a standard drained triaxial test on speswhite kaolin described by Al-Tabbaa (1987) are considered.

The time-integration of the finite-strain Cam-clay is performed using a Backward Euler scheme and a return-map algorithm, leading to the stable and accurate procedure discussed in (Callari et al., 1998).

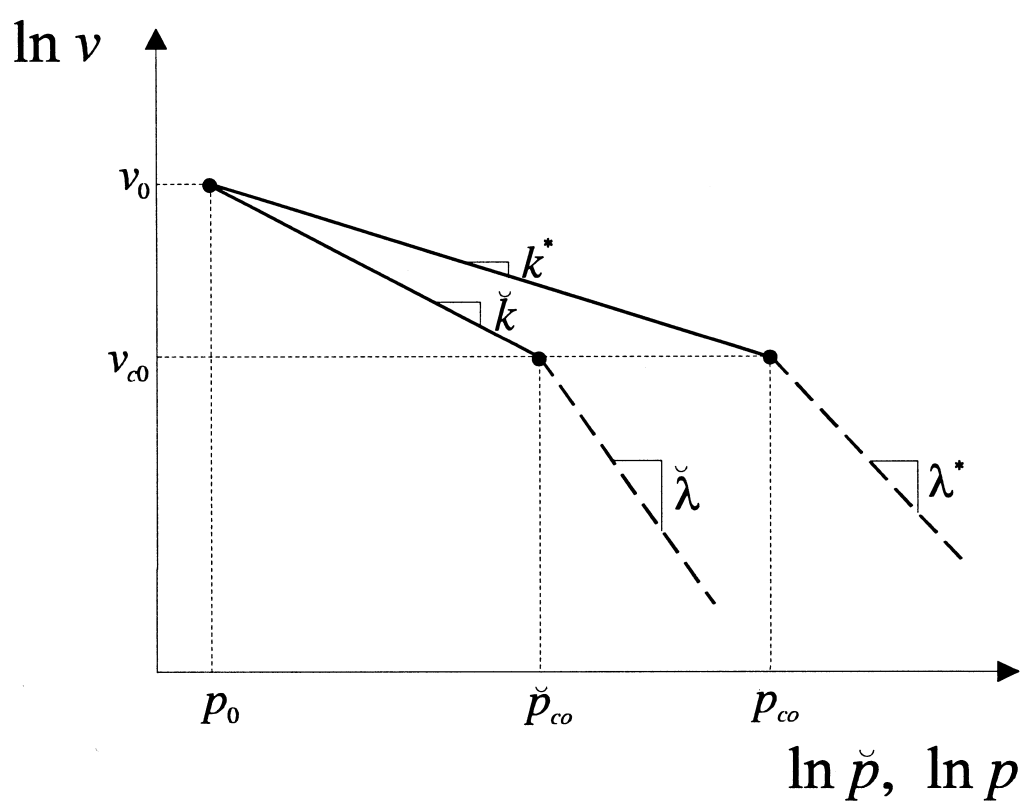

Fig. 11. Initial preconsolidation pressure in terms of Kirchhoff $\left(\breve{p}_{c 0}\right)$ and Cauchy $\left(p_{c 0}\right)$ stresses.

\$These constitutive relations rather than the "Kirchhoff" ones, are here required; in factor, $v_{c 1}$ is the specific volume corresponding to the Cauchy unit pressure. 


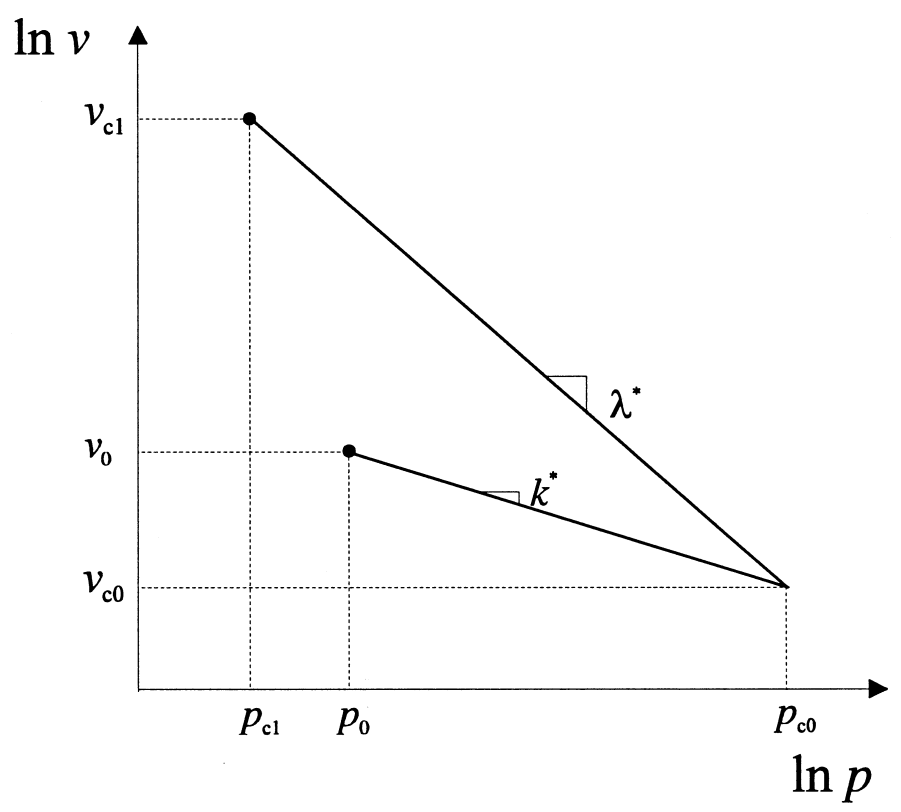

Fig. 12. Relation between the initial specific volume $\left(v_{0}\right)$ and the specific volume $\left(v_{c 1}\right)$ of a sample normally consolidated up to a unit pressure $\left(p_{c 1}\right)$.

Material parameters adopted in the numerical simulations are obtained from experimental data available in the literature, as described in the following.

\section{VI.1. Material parameters}

In (Al-Tabbaa, 1987), the "Cauchy" bilogarithmic indexes (see Section II.2) of speswhite kaolin are evaluated as $k^{*}=0.013$ and $\lambda^{*}=0.085$. Hence, recalling relations (5.19), the correspondent values of the "Kirchhoff" bilogarithmic indexes are computed as $k=0.013$ and $\check{\lambda}=0.093$.

The secant shear stiffness moduli, measured in the unloading-reloading phase of undrained triaxial tests on speswhite kaolin are reported by Houlsby (1985). In particular, the ratio $\mu_{u} / p$ between this modulus and the effective pressure varies within the interval $60 \div 110$. Hence, being $\alpha=\mu_{u} / p_{0}$ (see Section V.3), the elastic parameter $\alpha$ is to be chosen in this interval.

According to Al-Tabbaa (1987), the specific volume of a speswhite kaolin sample normally consolidated up to $p=1 \mathrm{kPa}$ is $v_{c 1}=3.20$. The slope of the critical state line is estimated as $M=0.9$. Still in (Al-Tabbaa, 1987), it is observed that the following intervals of $v_{c 1}$ and $M$ for speswhite kaolin are reported by other authors: $v_{c 1}=3.00 \div 3.65$ and $M=0.8 \div 0.9$.

Accordingly, in the numerical examples described in the following, the model parameters are set as:

$$
\check{k}=0.013 \quad \alpha=90 \quad \check{\lambda}=0.093 \quad M=\left\{\begin{array}{l}
0.8 \\
0.9
\end{array}\right.
$$


Furthermore, to represent the results also in terms of pressure-volume curves, $v_{c 1}=3.50$ is assumed.

\section{VI.2. Isotropic compression test}

In the test indicated as "test 1" in (Al-Tabbaa, 1987), a set of loading-unloadingreloading cycles are applied on an initially normally consolidated sample $\left(p_{0}=p_{c 0}\right.$ $=100 \mathrm{kPa}$ ). The bilogarithmic diagram of Fig. 13 shows the comparison between the experimental and the predicted data in terms of Cauchy pressure $p$ versus specific volume $v$. The initial specific volume $v_{0}=2.37$, given by relation $(5.43)$, is used to represent the numerical data.

The good agreement between model predictions and experimental data is apparent.

\section{VI.3. Triaxial compression test}

The drained triaxial test indicated as "test 13" in (Al-Tabbaa, 1987) is performed on a normally consolidated sample $\left(p_{0}=p_{c 0}=300 \mathrm{kPa}\right)$. When the deviatoric stress $q=120 \mathrm{kPa}$ is reached, the sample is unloaded. In Figs 14 and 15, the obtained stressstrain curves $q / p-\theta$ and $q / p-\varepsilon_{q}$ are plotted, respectively. Computations are repeated for values of $M$ belonging to the experimentally evaluated interval $M=0.8 \div 0.9$. The figures point out a satisfying agreement between predicted and observed data indicating $M=0.8$ as the most appropriate slope for the critical state line.

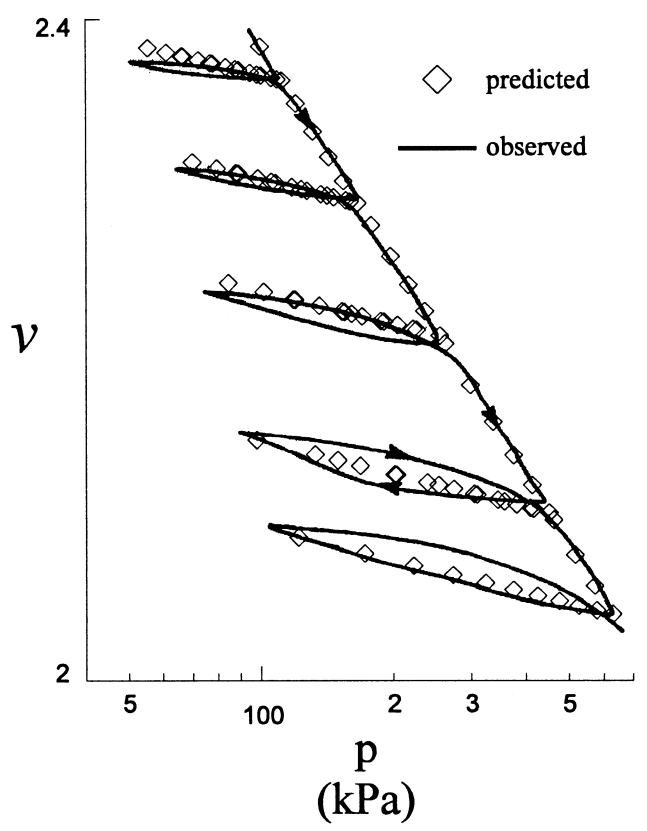

Fig. 13. Isotropic compression test on speswhite kaolin. Pressure $(p)$ vs. specific volume $(v)$. Model predictions and experimental data (Al-Tabbaa, 1987). 


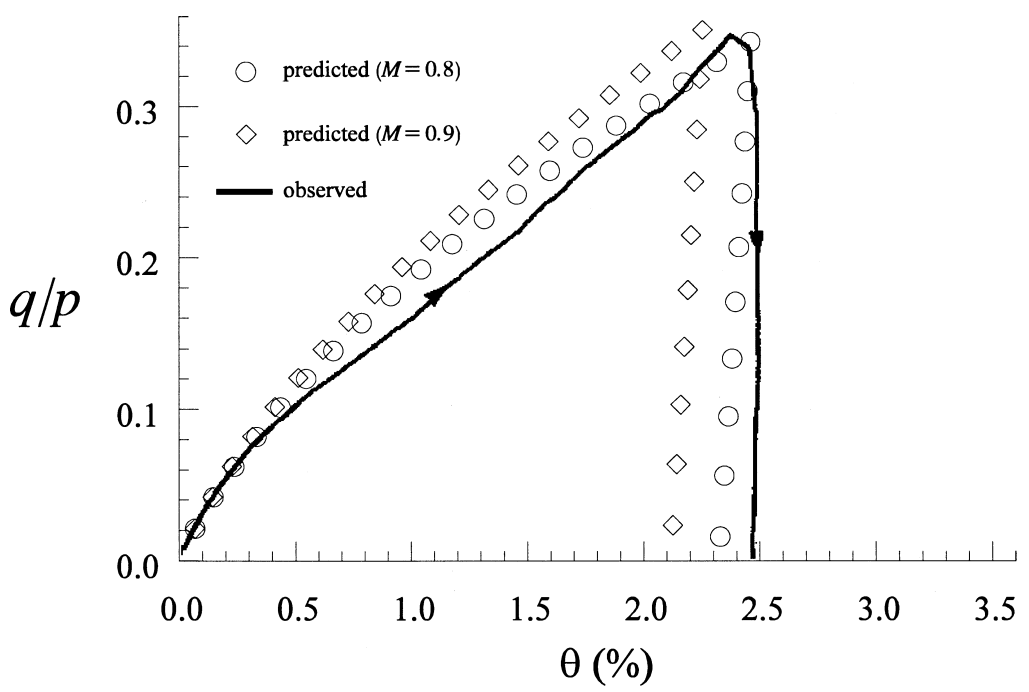

Fig. 14. Drained triaxial test on normally consolidated speswhite kaolin. Ratio $q / p$ vs. volumetric strain $\theta$. Model predictions and experimental data (Al-Tabbaa, 1987).

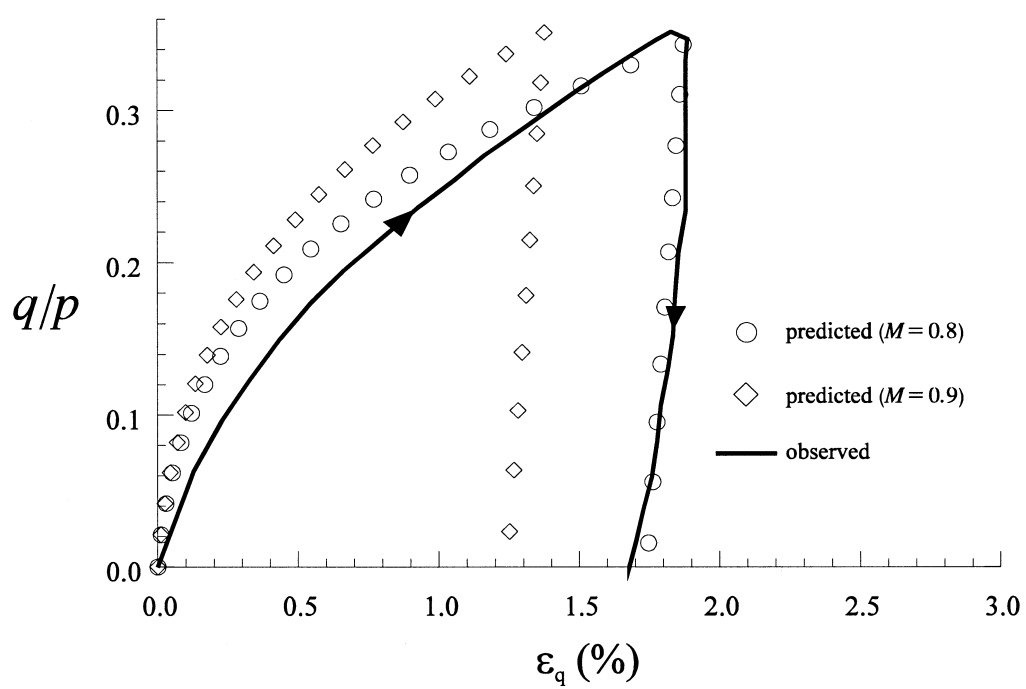

Fig. 15. Drained triaxial test on normally consolidated speswhite kaolin. Ratio $q / p$ vs. deviatoric strain $\varepsilon_{q}$. Model predictions and experimental data (Al-Tabbaa, 1987).

\section{CONCLUSIONS}

It is well-known that inelastic effects are major phenomena in the overall behavior of geomaterials. Moreover, experimental investigations and an accurate definition of kinematical aspects show that the infinitesimal-strain hypothesis is inadequate to the constitutive modelling of soft clays. 
Following these considerations, the elasto-plastic theory based on the multiplicative decomposition of the deformation gradient seems to be an effective framework for the constitutive description of soft clays. In particular, the extension of the elastic infinitesimal-strain energy proposed by Houlsby (1985) to the finite-strain regime combined with the Modified Cam-clay yield criterion (Roscoe and Burland, 1968) expressed in terms of Kirchhoff stresses leads to a model that is acceptable from a formal and a physical point of view. In fact, due to the assumed elastic potential, the shear tangent stiffness increases with pressure; hence, unlike other formulations, this realistic response is obtained using a conservative stress-strain relation. Furthermore, the adoption of bilogarithmic pressurevolume relations corrects the unrealistic predictions typical of traditional logarithmic isotropic compression laws.

The model calibration can be easily performed through the comparison of its constitutive laws in terms of Kirchhoff stress with the analogous relations in terms of Cauchy stress. The same comparison also points out some peculiarities of the elasto-plastic decomposition of volumetric strain.

Numerical simulations of laboratory tests have shown the model ability in reproducing some experimental data available in the literature.

\section{REFERENCES}

Al-Tabbaa, A. (1987) Permeability and stress-strain response of speswhite kaolin. Ph.D. thesis, University of Cambridge.

Atkinson, J. H. and Bransby, P. L. (1978) The Mechanics of Soils: An Introduction to Critical State Soil Mechanics. McGraw Hill, London.

Borja, R. I. (1991) Cam-clay plasticity, Part II: implicit integration of constitutive equation based on a non-linear elastic stress predictor. Comput. Methods Appl. Mech. Engrg. 88, 225-240.

Borja, R. I. and Lee, S. R. (1990) Cam-clay plasticity, Part 1: implicit integration of elasto-plastic constitutive relations. Comput. Methods Appl. Mech. Engrg. 78, 49-72.

Borja, R. I. and Tamagnini, C. (1995) Finite deformation theory for a Cam-clay model, Proc. 5th Numerical Models in Geomechanics (NUMOG V), Balkema, 3-8.

Borja, R. I. and Tamagnini, C. (1998) Cam-clay plasticity, Part III: extension of the infinitesimal model to include finite strains. Comput. Methods Appl. Mech. Engrg. 155, 73-95.

Borja, R. I., Tamagnini, C. and Amorosi, A. (1997) Coupling plasticity and energy-conserving elasticity models for clays. J. Geotech. and Geoenviromental Engrg. ASCE 123(10), 948-957.

Butterfield, R. (1979) A natural compression law for soils (an advance on $e-\log p^{\prime}$ ). Géotechnique 29, 469-480.

Callari, C., Auricchio, F. and Sacco, E. (1996) A return map algorithm for a finite-strain cam-clay plasticity model. Joint Conf of Italian Group of Comp. Mech. and Ibero-Latin American Association of Comp. Meth. in Eng., Padova.

Callari, C., Auricchio, F. and Sacco, E. (1997) Finite-element implementation of a finite-strain Cam-clay model. Proc. 5th Int. Conf on Computational Plasticity: Fundamentals and Applications (COMPLAS V), CIMNE, Vol. 2, Barcelona, pp. 1649-1656.

Callari, C., Auricchio, F. and Sacco, E. (1998) An implicit integration technique for the finite-element implementation of a finite-strain Cam-clay model, submitted for publication.

Chadwick, P. (1976) Continuum Mechanics. John Wiley and Sons, New York.

Coleman, B. D. and Gurtin, M. E. (1967) Thermodynamics with internal state variables. J. Chem. Phys. 47, 597613.

Coulomb, C. A. (1776) Essai sur une application des règles de maxims et minims à quelques problèmes de statique, relatifs à l'architecture. Mèm. Acad. R. Sci. 7, 343 .

Drucker, D. C. (1953) Limit analysis of two and three dimensional soil mechanics problems. J. Mech. Phys. Solids 1, 217-226.

Drucker, D. C., Gibson, R. E. and Henkel, D. J. (1957) Soil mechanics and workhardening theories of plasticity. Trans. ASCE 122, 338-346.

Drucker, D. C. and Prager, W. (1952) Soil mechanics and plastic analysis or limit design. Quart. Appl. Math. 10, 157-165.

Gens, A. and Potts., D. M. (1988) Critical state models in computational geomechanics. Engrg. Comp. 5, 178197. 
Groen, A. E. (1995) Two elastoplastic models for the behaviour of soils, report nr. 03.21.0.31.12, TU Delft.

Groen, A. E., de Borst, R. and van Eckelen, S. A. M. (1995) An elastoplastic model for clay. Formulation and algorithmic aspects. Proc. 5th Numerical Models in Geomechanics (NUMOG V), Balkema, pp. $27-32$.

Gurtin, M. E. (1981) An introduction to Continuum Mechanics. Academic Press, Orlando, Florida.

Hashash, Y. M. A. and Whittle, A. J. (1992) Integration of the modified Cam-clay model in non-linear finite element analysis. Computers and Geotechnics 14, 59-83.

Hashiguchi, K. (1995) On the linear relations of $V-\ln p$ and $\ln v-\ln p$ for isotropic consolidation of soils. Int. J. Numer. Analyt. Methods Geomechanics 19, 367-376.

Hashiguchi, K. and Ueno, M. (1977) Elastoplastic constitutive laws of granular materials. Proc. 9th Int. Conf SMFE, Specialty Session 9, Tokio, pp. 73-82.

Hill, R. (1951) The Mathematical Theory of Plasticity, Oxford University Press, Oxford, U.K.

Houlsby, G. T., Wroth, C. P. and Wood, D. M. (1982) Predictions of the results of laboratory tests on a clay using a critical state model. Proc. Int. Workshop on Constitutive Behaviour of Soils, Balkema, Rotterdam, pp. 99-121.

Houlsby, G. T. (1985) The use of a variable shear modulus in elastic-plastic models for clays. Comput. Geotechn. 1, 3-13.

Lee, E. H. (1969) Elastic-plastic deformation at finite strains. J. Appl. Mech. 36, 1-6.

Lee, E. H. and Liu, D. T. (1967) Finite strain elastic-plastic theory particularly for plane wave analysis. J. Appl. Phys. 38.

Marsden, J. E. and Hughes, T. J. R. (1983) Mathematical Foundations of Elasticity. Prentice Hall Englewood Cliffs, New Jersey.

Martin, C. L., Favier, D. and Suéry, M. (1997) Viscoplastic behaviour of porous metallic materials saturated with liquid. Part I: Constitutive equations. Int. J. Plasticity 13(3), 215-235.

Ogden, R. W. (1984) Nonlinear Elastic Deformations. Ellis Horwood, West Sussex, England.

Podio-Guidugli, P. and Vergara Caffarelli, G. (1991) Extreme elastic deformations. Arch. Rational Mech. Anal. 115, 311-328.

Rankine, W. J. M. (1857) On the stability of loose earth. Phil. Trans. R. Soc. 147, 9-27.

Roscoe, K. H. and Burland, J. B. (1968) On the generalized stress-strain behaviour of 'wet' clay. Engineering Plasticity, pp. 535-609. Cambridge Univ. Press, Cambridge.

Roscoe, K. H. and Schofield, A. N. (1963) Mechanical behaviour of an idealized 'wet' clay. Proc. 2nd Eur. Conf SMFE, Wiesbaden, Vol. 1, pp. 47-54.

Saada, R. A., Bonnet, G. and Bouvard, D. (1996) Thermomechanical behavior of casting sands: Experiments and elastoplastic modeling. Int. J. Plasticity, 12(3).

Schofield, A. N. and Wroth, C. P. (1968) Critical State Soil Mechanics. McGraw-Hill.

Shield, R. T. (1955) On Coulomb's law of failure in soils. J. Mech. Phys. Solids 4(1), 10-16.

Simo, J. C. (1992) Algorithms for static and dynamic multiplicative plasticity that preserve the classical return mapping schemes of the infinitesimal theory. Comput. Methods Appl. Mech. Engrg. 99, 61-112.

Simo, J. C. and Meschke, G. (1993) A new class of algorithms for classical plasticity extended to finite strains. Application to geomaterials. Computational Mechanics 11, 253-278.

Wroth, C. P. (1975) The predicted performance of soft clay under a trial embankment loading based on the Camclay model. In Finite Elements in Geomechanics, pp. 191-208. John Wiley and Sons, London.

Wroth, C. P. and Houlsby, G. T. (1980) A critical state model for predicting the behaviour of clays. In Workshop on Limit Equilibrium, Plasticity and Generalized Stress-strain in Geotechnical Engineering, pp. $592-627$.

Wroth, C. P. and Houlsby, G. T. (1985) Soil mechanics-property characterization and analysis procedures. Proc. 11th Int. Conf SMFE, San Francisco, Vol. 1, pp. 1-55.

Wood, D. M. (1990) Soil Behaviour and Critical State Soil Mechanics. Cambridge University Press, Cambridge.

Zytinsky, M., Randolph, M. K., Nova, R. and Wroth, C. P. (1978) On modelling the unloading-reloading behaviour of soils. Int. J. Numer. Analyt. Methods Geomechanics 2, 87-93. 